\title{
Out-of-equilibrium dynamical fluctuations in glassy systems
}

\author{
C. Chamon ${ }^{\text {a) }}$ \\ Department of Physics, Boston University, 590 Commonwealth Avenue, Boston, Massachusetts 02215 \\ P. Charbonneau \\ Department of Chemistry and Chemical Biology, Harvard University, 12 Oxford Street, Cambridge, \\ Massachusetts 02138 \\ L. F. Cugliandolob) \\ Laboratoire de Physique Théorique, Ecole Normale Supérieure, 24 rue Lhomond, 75231 Paris Cedex 05, \\ France and Laboratoire de Physique Théorique et Hautes Energies, Jussieu, 4 Place Jussieu, \\ 75252 Paris Cedex 05, France \\ D. R. Reichman ${ }^{\text {c) }}$ \\ Department of Chemistry and Chemical Biology, Harvard University, 12 Oxford Street, Cambridge, \\ Massachusetts 02138 \\ M. Sellitto ${ }^{\text {d) }}$ \\ The Abdus Salam International Centre for Theoretical Physics, Strada Costiera 11, 34100 Trieste, Italy
}

(Received 19 January 2004; accepted 1 September 2004)

\begin{abstract}
In this paper we extend the earlier treatment of out-of-equilibrium mesoscopic fluctuations in glassy systems in several significant ways. First, via extensive simulations, we demonstrate that models of glassy behavior without quenched disorder display scalings of the probability of local two-time correlators that are qualitatively similar to that of models with short-ranged quenched interactions. The key ingredient for such scaling properties is shown to be the development of a criticallike dynamical correlation length, and not other microscopic details. This robust data collapse may be described in terms of a time-evolving "extreme value" distribution. We develop a theory to describe both the form and evolution of these distributions based on a effective $\sigma$ model approach. (C) 2004 American Institute of Physics. [DOI: 10.1063/1.1809585]
\end{abstract}

\section{INTRODUCTION}

The dynamics of glassy systems is extremely slow, both near and far from equilibrium. ${ }^{1}$ Until recently, experiments, numerical studies, and theory focused on the study of averaged global, or bulk, dynamical quantities that demonstrate the nonequilibrium character of the glassy state. Interest is now shifting to the study of local quantities embodied in intermittent fluctuations and dynamic heterogeneities. The recent development of powerful experimental techniques ${ }^{2-10}$ and the use of extensive numerical simulations have started to yield valuable information about the dynamics of supercooled liquids ${ }^{11-19}$ and glassy systems ${ }^{20,21}$ at a mesoscopic scale. A detailed analysis of temporal fluctuations in the evolution of global observables allows one to distinguish between intermittent and continuous dynamics, the former being associated with sudden, large rearrangements. ${ }^{6,9,10}$ In addition, the study of run-to-run fluctuations in systems of mesoscopic size yields complementary information. ${ }^{21}$ The insight obtained from these studies is useful in identifying the most relevant rearrangements that take place during evolution and thus may lead to a better understanding of the mechanism leading to glassy arrest.

\footnotetext{
a)Electronic mail: chamon@buphy.bu.edu

b)Electronic mail: leticia@1pt.ens.fr

c) Electronic mail: reichman@chem.harvard.edu

d)Electronic mail: sellitto@ictp.trieste.it
}

The behavior of global dynamic correlations in macroscopic glassy systems is rather well described analytically with an extension of the mode-coupling theory to the low temperature regime. ${ }^{22,23}$ This approach is also attractive since, via its connection to mean-field models with quenched disorder, it allows for an interpretation of dynamic arrest and slow dynamics in terms of the geometric properties of a freeenergy landscape. ${ }^{24-27}$ However, it is not clear how to describe temporal and spatial fluctuations within this approach. Another picture of glassy dynamics is based on kinetically constrained models with trivial static interactions ${ }^{28-30}$ and is successful in describing aging effects and other glassy features $^{31}$ as has been recently reviewed. ${ }^{32}$ Moreover, dynamic heterogeneities also find a natural interpretation within these models. ${ }^{33,34}$ The connection between the microscopic interacting real system and the noninteracting kinetically constrained model is, however, phenomenological, as the facilitation rules are based on a coarse-grained description of the liquid dynamics. Other phenomenological descriptions do not even have a real-space interpretation. Thus, one of the more interesting and open theoretical problems at present is how to derive the observed properties of medium size and large-scale fluctuations that are possibly associated with cooperative and correlated rearrangements of the microscopic constituents (particles, spins, etc.) from a microscopic model. $^{34}$

In this paper we focus on particular aspects of the non- 
equilibrium dynamics near or below the onset of glassy arrest. Some of our arguments and numerical analysis can be simplified to capture the (stationary) slow dynamics of supercooled liquids as a particular case. The purpose of this paper is threefold. First, we test whether the scenario proposed in Refs. 20, 21, and 35 for spatially heterogeneous glassy dynamics applies to models without quenched disorder such as kinetically constrained spin models. Second, we show that several features of the probability distributions functions (PDFs) of fluctuating two-time correlations, namely, time scalings and functional forms, are common to many glassy systems. Third, we discuss an analytic approach developed to describe the scaling form and time evolution of these PDFs. This approach is based on the arguments put forward in Refs. 20 and 35, and on an analogy with the study of the order-parameter fluctuations in critical phenomena, ${ }^{36}$ where one can describe universality classes in terms of the PDFs of global fluctuations. The latter is similar in spirit to the work of Rácz and collaborators on interfacial growth problems ${ }^{37}$ and Bramwell et al. on the $X Y$ model. ${ }^{38}$

Even if, strictly speaking, there is no liquid-glass dynamic phase transition, glassy dynamics present some aspects of critical dynamics. Among others, a dynamic correlation length defined from the fluctuations of the local twotime correlations shows an apparent divergence at long times. ${ }^{21,39-42}$ In the critical situation, one expects that the symmetries of the problem constrain the form and scaling of the PDFs of fluctuating dynamical quantities. A natural way to attempt an analytic approach to these fluctuations is then to construct an effective $\sigma$ model that respects these symmetries. This approach has been advocated in Refs. 20, 21, and 35 . Here we extend it to propose a model that describes not only the time scaling of the PDFs but also their functional form.

The plan of the paper is as follows. In Sec. II we summarize the main properties of glassy dynamics as displayed by the decay of global correlation functions. In Sec. III we present a short review of experiments that focus on the measurement of dynamic fluctuations. In Sec. IV we introduce the models that we study numerically; these are a modification of the Fredrickson-Andersen (FA) model due to Graham, Piché, and Grant ${ }^{43}$ and the Edwards-Anderson (EA) spin glass. ${ }^{44}$ In Sec. V we present the PDFs for fluctuating two-time correlations. We demonstrate that very similar PDFs are obtained from facilitated Ising models without quenched random interactions and the disordered spin-glass problem. These forms also resemble strongly the experimental data of Cipelletti et al. ${ }^{10}$ for colloidal suspensions. We describe these PDFs with an evolving extreme value form that we later argue can be justified from the sigma-model approach. In Sec. VI we introduce the theoretical modeling of these two-time dependent PDFs. Finally, in Sec. VII we present our conclusions. Appendix A discusses the definition of a Gumbel distribution and some of its properties. The aim of Appendix B is to identify which features of the PDFs are truly due to glassy dynamics. To this end we analyze the stochastic dynamics (with no facilitation rule) of a finite system of independent spins on a lattice.

\section{NONEQUILIBRIUM RELAXATION IN GLASSY SYSTEMS}

Glassy systems show slow nonexponential dynamical relaxation. This is demonstrated by the extremely sluggish decay of one-time observables such as the energy density, the structure factor, etc. In many experimental cases, one-time observables can be considered to be approximately constant within the time window explored. Thus, observables that depend on only one time are not sufficient to characterize the nonequilibrium relaxation of a glassy system in its full richness; they do not signal one of the hallmarks of glassy systems, namely aging phenomena. Aging is partially described by the decay of two-time global correlation functions:

$$
C\left(t, t_{w}\right) \equiv \mathcal{N}^{-1}\left\langle O(t) O\left(t_{w}\right)\right\rangle,
$$

with $O(t)$ the global observable of interest and $\mathcal{N}$ a two-time dependent normalization that enforces $C(t, t)=1$. The angular brackets denote an average over different thermal histories of the system. In a model with quenched disorder, e.g., the EA spin glass, one averages the global correlation over different realizations of the interactions. Sometimes a coarse graining in time using a short time window around the observation instant is also implemented. It is important to note that for a sufficiently large system, the quantity,

$$
O(t) O\left(t_{w}\right) / \mathcal{N},
$$

does not fluctuate and the result obtained for a single thermal history (and a single realization of disorder) coincides with the averaged one, as long as times are long, but not so long as to exhibit recovery of equilibrium behavior.

As already mentioned, one-time observables evolve very slowly in the glassy phase of real systems. Still, some models used to capture glassy dynamics have a faster evolution of one-time quantities. In these cases, it is convenient to study the evolution of the connected correlations defined as in Eq. (1) where

$$
O(t) \rightarrow O(t)-\langle O(t)\rangle \text {. }
$$

By the very definition of aging, older systems relax in a slower manner than younger ones. One defines the age of a system as the time spent in the phase under study. A way to characterize the aging properties is to monitor the time evolution of the two-time correlation defined in Eq. (1) or the two-time function in Eq. (2). Well below $T_{g}$ (conventionally defined as the value at which the relaxation time reaches 100s) the system is in a "phase" that we call "glassy." At such low temperatures the correlations depend explicitly on $t_{w}$ and, within the experimentally accessible time-window, do not show a tendency towards decay to equilibrium behavior, that is to say an independence from $t_{w}$. Close to glassy arrest one observes "interrupted aging," namely, a dependence on the age of the system until observations reach the equilibration time $\left(t_{w}>t_{\mathrm{EQ}}\right)$ where the dynamics crosses over to an equilibrium one.

The correlations of Eq. (1) have been studied experimentally in a number of glassy materials. In spin systems the natural choice for $O(t)$ is the fluctuation of the global magnetization, ${ }^{45}$ while in colloidal suspensions it is the intensity of scattered light. ${ }^{10,46-48}$ Many numerical studies of a 
large variety of models have focused on the analysis of the closely related intermediate scattering function. On the analytical side, the study of mean-field disordered models such as the $p$-spin model yielded several features of the aging behavior seen in simulations and experiments. ${ }^{23,24}$ Connected global correlations in several kinetically constrained spin systems have also been studied yielding similar results. ${ }^{31,32}$

In structural glasses, a pictorial explanation of aging may be put forward by imagining that each particle sees a cage made of its neighbors. When $\tau$ is short compared to a characteristic time $\tau_{0}\left(t_{w}\right)$, each particle rattles within its cage, decorrelation is characterized only by thermal fluctuations, and correlations decay in a stationary manner from unity at equal times to a plateau $q_{\mathrm{EA}}(T)$ seen on a logarithmic scale. When $\tau$ increases, the motion of the particles destroys the cages and the structure of the system relaxes. The waiting-time dependence implies that the cages become stiffer as time evolves. The motion of a tagged particle observed with confocal microscopy demonstrated this scenario. ${ }^{6}$ Upon examination of the value of the displacement reached at the maximum separation time, however, one notices that motion occurs only on length scales of the order of the radius of a particle. This means that the structural relaxation occurs without large scale structural rearrangement and with essentially no exchange of particles within the bulk of the sample. The same observation applies to the dynamics of granular matter under external forcing. ${ }^{49}$

The existence of a well-defined cage translates into the appearance of a plateau in the decay of the two-time correlations. The presence of a plateau in the correlation function means that there is a macroscopic time interval in which the correlation (when plotted in log-log scale) can be approximated by a (nonzero) constant. In this case the two-time correlation can be scaled in a satisfactory way by using a separation in fast and slow regimes: ${ }^{23,24,50}$

$$
\begin{aligned}
C\left(t, t_{w}\right) & =C_{F}\left(t-t_{w}\right)+C_{S}\left(t, t_{w}\right) \\
& =f_{F}\left(\frac{h_{F}(t)}{h_{F}\left(t_{w}\right)}\right)+C_{S}\left(t, t_{w}\right),
\end{aligned}
$$

with $h_{F}(t)=e^{t / \tau_{F}}$ and $\tau_{F}$ a characteristic time scale. The first term describes the stationary approach to the plateau, the second term the $t_{w}$-dependent departure from it. The lightscattering study of a colloidal system of Viasnoff and co-workers ${ }^{47}$ shows a very clear plateau separating the two regimes. However, a clear-cut plateau is not always visible in numerical simulations and experiments.

In most cases studied so far the second (aging) decay is characterized by a similar form

$$
C_{S}\left(t, t_{w}\right)=f_{S}\left(\frac{h_{S}(t)}{h_{S}\left(t_{w}\right)}\right),
$$

with $h_{S}(t)$ a system-dependent monotonic function. A typical and well-understood example in which the slow part of the decay is represented by this form is the case of systems undergoing domain growth. In this case, for long and wellseparated times, $h_{S}(t)$ is the typical size of the domains at time $t$. Other examples include the relaxation of the incoherent scattering function in Lennard-Jones mixtures and the result of light-scattering measurements in colloidal suspensions. ${ }^{10,47,48}$ Monte Carlo data for the two-time spinspin correlations in the $3 d$ EA model can be described with such a two-step relaxation and $h_{S}(t)=t{ }^{51}$ Similarly, the magnetization correlations in the insulating spin glass studied by Hérisson and Ocio ${ }^{45}$ are described by a two-step relaxation. In this study, an enhanced power law $h_{S}(t)$ $=e^{\ln ^{a} t / t_{0}}$ yields the best fit of the experimental data. We shall see below that, for the times that one can reach with a numerical simulation, the kinetically constrained models defined in Sec. IV either do not show a plateau in their connected correlations, or show several plateaus. Moreover, it is very difficult to identify a single function $h_{S}(t)$ that describes the slow decay. This might be due to the fact that the slow decay involves several correlation scales that are still not well separated during numerical times.

\section{HETEROGENEOUS DYNAMICS}

The existence of dynamic heterogeneities in supercooled liquids and glasses has been suggested on the basis of experiments performed using many techniques, ${ }^{2}$ two of them are the confocal microscopy and light scattering. We discuss these results in some detail since we shall compare our numerical simulations to them.

Confocal microscopy allows one to reconstruct the trajectory of each particle in a colloidal suspensions made of several thousand particles., ${ }^{3,4}$ Kegel and van Blaaderen ${ }^{3}$ found a non-Gaussian distribution of particle displacements in a dense system of colloidal hard spheres and they associated this large distribution to the presence of dynamical heterogeneities. Later, Courtland and Weeks found that mobile (and immobile) particles cluster in regions of lower (higher) density than average in the glassy phase of a colloidal system. ${ }^{6}$

The "time-resolved light-scattering technique" (Ref. 10) has been developed with the aim of testing the fluctuations in time of the intensity-intensity correlations in samples of medium size. The existence of intermittency in measurements of global quantities may be another manifestation of heterogeneous dynamics. Temporal fluctuations in colloidal suspensions have been studied in this way. Interestingly enough, Cipelletti's group showed that aging samples, such as concentrated colloidal gels, have an intermittent dynamics leading to negatively skewed distributions of the two-time intensity-intensity correlations when the time lag is shorter than the relaxation time and Gaussian distributions when the time lag approaches the relaxation time. They also showed that simpler systems with no glassy features, such as Brownian colloidal samples, have a continuous relaxation with a Gaussian distribution of the same quantities for all time lags explored. The same trend of time resolved PDFs of particle displacements was demonstrated by Courtland and Weeks using confocal microscopy. ${ }^{6}$ It is interesting to note that these experiments show that significant fluctuations occur even if the time lag $\tau$ is very short compared to $t_{w}$.

Evidence for intermittency in the global voltage signal noise in a solidlike colloidal glass and a polymer glass has also been presented recently. ${ }^{9}$ While the samples are out of 
equilibrium the voltage time series has bursts with very large amplitudes. These bursts become rarer with the sample age in the sense that their amplitude decreases and the time elapsed between two consecutive bursts increases. When aging stops the bursts disappear from the noise signal. The waiting-time dependent PDF $\rho(V)$ constructed by dividing time in intervals of duration $\tau$ is clearly negatively skewed at short times and progressively approaches a Gaussian distribution when the age of the system increases. ${ }^{9}$

On the numerical side, most studies have focused on the supercooled liquid phase of binary mixtures ${ }^{11-16}$ and polymer melts ${ }^{17}$ and the heterogeneities have been studied by tagging each particle and classifying them according to their mobility during a selected time interval. Some recent papers treat the glassy phase of the Lennard-Jones system in a similar way. ${ }^{19}$ Following the dynamics of individual particles is very useful to get an intuitive understanding of the evolution of the system, but it is hard to use as a direct input in a theoretical approach. In order to develop such a description, it is much more convenient to use the coarse-grained twotime quantities ${ }^{20,35}$ that we shall define in Sec. V. In this setting, a heterogeneity is a region in the sample that relaxes very differently from the bulk. Clearly, the heterogeneities are not static and they appear and disappear as dynamic fluctuations.

\section{THE MODELS}

We shall focus on several spin models that describe and capture different aspects of glassy systems. We define them below.

\section{A. Kinetically constrained model}

\section{A variation on the Fredrickson-Andersen model}

The first model we study is a kinetically constrained spin system with trivial equilibrium properties. It is a simple variation of the Fredrickson-Andersen (FA) mode ${ }^{28}$ due to Graham, Piché, and Grant. ${ }^{43} N$ Ising spins, $s_{i}= \pm 1, i$ $=1, \ldots, N$, occupy the vertices of a regular cubic lattice in $d$ dimensions. The energy is given by

$$
E=-\frac{h}{2} \sum_{i=1}^{N} s_{i} .
$$

Thus there are no static interactions between the spins but an external field of strength $h$ is applied. The system is in contact with a heat bath at temperature $T$ that, for convenience, we shall measure in units of the external field $h$. This model does not have a static transition and at equilibrium the averaged magnetization $m=\Sigma_{i}\left\langle s_{i}\right\rangle / N$ is given by

$$
m=\tanh \frac{h}{2 k_{B} T} .
$$

The nontrivial evolution of this noninteracting system arises due to the constrained dynamics imposed on the spins. At each time step a spin $s_{k}$ that is chosen at random among the ensemble is allowed to flip with a generalized heat bath rule. If at least $p$ of its $z=2 d$ neighbors point down, i.e., opposite to the preferred direction, the move is accepted with unit probability if $\Delta E<0$ and with probability $e^{-\Delta E / k_{B} T}$ if $\Delta E$
$>0 . \Delta E=E_{f}-E_{i}$, with $E_{f}$ the total energy after the move and $E_{i}$ the total energy before the move. In this case, $\Delta E$ $=\left(-s_{k}^{f}+s_{k}^{i}\right) / 2=s_{k}^{i}$. Otherwise, the attempted flip is rejected. The parameter $p$ defines the facilitated rule. For instance, a three-site rule requires a minimum of three neighbors to oppose the field direction. As usual, time units are defined via the number of attempts to flip randomly chosen spins in the sample. The facilitation rule satisfies detailed balance. Thus the system evolves towards the equilibrium configuration, although it may do so in a very slow manner. The larger the value of $p$ the slower the resulting dynamics.

Typically, one is interested in following the relaxational dynamics of a random initial condition in which each spin points in the up or down direction with probability one half. This mimics a rapid quench from infinite temperature to the working temperature $T$. The dynamics tend to order the system in the up direction. However, as time passes, it is harder to choose a spin with sufficient number of neighbors pointing down. This induces a dynamic slowing down, and below $T$ $\sim 0.5$ (in $d=3$ and using $p=3$ ) or $T \sim 0.35$ (in $d=2$ and using $p=2$ ) the system does not manage to get close to the equilibrium configuration. The out-of-equilibrium relaxation below this crossover temperature resembles that of supercooled liquids. ${ }^{52}$

Importantly, these models do not contain quenched disorder. The absence of quenched disorder and the local, nonmean-field nature of the relaxation strongly suggests that such models are relevant for understanding dynamics of supercooled liquids. The existence or absence of a genuine glass transition is not important for our purposes. Indeed, as it has been recently shown, rigorously, in some cases these models do not have a dynamic phase transition in the thermodynamic limit. ${ }^{53}$ Nevertheless, the evolution of a large finite system at long but finite times shares some keynote properties with many glassy systems, including systems that have a finite temperature dynamic transition, such as spinglass models that undergo one-step replica symmetry breaking. Thus, the study of facilitated models in the context presented here is of even broader importance than just the modeling of supercooled liquids.

\section{B. The Edwards-Anderson spin glass}

A disordered model that we shall briefly discuss here is the standard non-mean-field model for spin-glasses, the finite dimensional Edwards-Anderson model. ${ }^{44}$ The energy function is highly nontrivial in this case,

$$
E_{J}=-\sum_{\langle i j\rangle} J_{i j} s_{i} s_{j},
$$

where the spins are Ising variables that occupy the vertices of a cubic $d$-dimensional lattice, the sum runs over nearest neighbors, and the coupling strengths are random and chosen from a bimodal or Gaussian probability distribution with zero mean and variance $\left[J_{i j}^{2}\right]=1 /(2 z)$. The square brackets denote an average over the random exchanges. This model is said to be disordered due to the quenched randomness introduced via the exchanges. The static properties are still under debate. It is believed that in $3 d$ the Edwards-Anderson 
model undergoes a static phase transition from a paramagnetic to a spin-glass phase at a finite value of $T$, which depends on the type of disorder used, but there is no consensus about the nature and properties of the static spin-glass phase. ${ }^{54}$ Dynamically, one may also follow the evolution of spin configurations from a random initial condition. The dynamics is, in this case, usual Monte Carlo with the heat bath rule determined by the energy function (8). The evolution of a sufficiently large system at low temperatures occurs out of equilibrium.

The local mesoscopic fluctuations of this model have been studied analytically and numerically in Refs. 20, 21, and 35 . We shall not reproduce these results here. In this manuscript we merely augment the results presented in Refs. 20,21 , and 35 to test the properties of fluctuations that have not been discussed in previous publications.

\section{FLUCTUATIONS}

In this section we introduce the quantities we shall focus on and we discuss the behavior of their PDFs as found for the kinetically constrained models and the $3 d$ EA spin glass.

\section{A. Spatial fluctuations}

Local fluctuations in an unperturbed system are monitored by studying

$$
\begin{aligned}
C_{r}^{\circ}\left(t, t_{w}\right) \equiv & \frac{1}{V_{r}} \sum_{x \in V_{r}} O(x, t) O\left(x, t_{w}\right) \\
& -\frac{1}{V_{r}} \sum_{x \in V_{r}} O(x, t) \frac{1}{V_{r^{\prime}} \sum_{x^{\prime} \in V_{r}} O\left(x^{\prime}, t_{w}\right),}
\end{aligned}
$$

where $V_{r}=\ell^{d}$ is a coarse-graining volume centered at the position $r$. This is a local connected function that evolves from a time-dependent value at equal times to zero at diverging time separations. Since it is convenient to work with a normalized two-time quantity at equal times, we define

$$
C_{r}\left(t, t_{w}\right) \equiv \frac{C_{r}^{\circ}\left(t, t_{w}\right)}{\sqrt{C_{r}^{\circ}(t, t) C_{r}^{\circ}\left(t_{w}, t_{w}\right)}} .
$$

With this definition the normalization itself is site dependent and might introduce spurious fluctuations. Another possibility is to normalize the local correlation with the global connected one

$$
C_{r}\left(t, t_{w}\right) \equiv \frac{C_{r}^{\circ}\left(t, t_{w}\right)}{\sqrt{C(t, t) C\left(t_{w}, t_{w}\right)}} .
$$

This normalization bounds the global correlation to be less than unity but it does not bound the local one; in particular, it does not enforce the local correlation to be one at equal times. In what follows, we present results obtained using definition (11) only.

For lattice spin models, the choice is

$$
\begin{aligned}
C_{r}^{\circ}\left(t, t_{w}\right) \equiv & \frac{1}{V_{r}} \sum_{i \in V_{r}} s_{i}(t) s_{i}\left(t_{w}\right) \\
& -\frac{1}{V_{r}} \sum_{i \in V_{r}} s_{i}(t) \frac{1}{V_{r}} \sum_{j \in V_{r}} s_{j}\left(t_{w}\right)
\end{aligned}
$$

and its normalized counterpart. For the EA model one easily verifies that, when the coarsegraining volume is sufficiently large, the local magnetization vanishes, $V_{r}^{-1} \sum_{i \in V_{r}} s_{i}\left(t_{w}\right)$ $=0$, and there is no need to use the connected functions above. For the modified FA model, the local magnetization does not vanish and we shall work with connected functions. In none of these expressions is there any averaging, apart from the coarse-graining in space.

With the local observables one can define a two-time dependent correlation length $\xi\left(t, t_{w}\right)$ via $^{21}$

$$
\begin{aligned}
A\left(\Delta ; t, t_{w}\right) \equiv & \frac{1}{V} \int d^{d} x O(\vec{x}, t) O\left(\vec{x}, t_{w}\right) O(\vec{x}+\vec{\Delta}, t) \\
& \times O\left(\vec{x}+\vec{\Delta}, t_{w}\right)
\end{aligned}
$$

and the normalized quantity

$$
\begin{aligned}
B\left(\Delta, t, t_{w}\right) & \equiv \frac{A\left(\Delta ; t, t_{w}\right)-A\left(\Delta \rightarrow \infty ; t, t_{w}\right)}{A\left(\Delta=0 ; t, t_{w}\right)-A\left(\Delta \rightarrow \infty ; t, t_{w}\right)} \\
& \sim e^{-\Delta / \xi\left(t, t_{w}\right)} .
\end{aligned}
$$

(We have made explicit the vectorial character of the positions $\vec{x}$ in $d>1$. In the case of spin problems it is convenient to use $O(\vec{x}, t)=\bar{s}_{i}(t)$, where the overline indicates a coarse graining in time over a time window centered on $t$.)

Depending on the relative value between the linear size of the coarse-graining volume $\ell$ and the two-time dependent correlation length $\xi$ we expect either:

(i) If $\ell$ is not much larger than $\xi\left(t, t_{w}\right)$ different cells in the system behave rather differently and the histogram of the quantities (10) leads to a nontrivial PDF whose form depends on the two times chosen, $t_{w}$ and $t$.

(ii) If $\ell \gg \xi\left(t, t_{w}\right)$ the coarse-graining averages over many independent cells and the PDF approaches a Gaussian form that, in the limit $\ell \sim L \rightarrow \infty$, has a vanishing variance and its average is given by the global value of the correlation.

As a consequence one expects to find similar fluctuations by taking a system with finite size and examining the behavior of the run-to-run fluctuations of the global two-time quantity (2). Indeed, if $L \gg \xi$ one falls in case (ii). Instead, if $L$ is of the order of $\xi$, case $i$ is found and the "mesoscopic fluctuations" should be nontrivial. This argument justifies the comparison of our approach to the ones in Refs. 9 and 10.

\section{B. Variant of the Fredrickson-Andersen model}

In this section we present the analysis of the dynamics of the kinetically facilitated model in $d=3$ choosing $p=3$ and then in $d=2$ for $p=2$. All the $3 d$ numerical data have been obtained using a cubic lattice of linear size $L=128$ lattice spacings at temperature $T=0.4$ and the $2 d$ data using a square lattice with $L=1000$ at temperature $T=0.3$. Coarsegrained data have been obtained using a cubic coarse- 


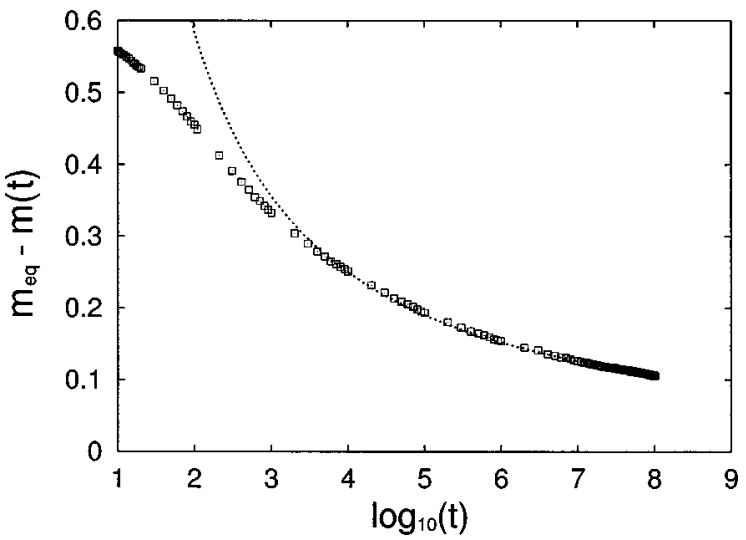

FIG. 1. Time evolution of the magnetization density in the modified FA model. $d=3, L=128, T=0.4$. The line is a fit to the curve given in Eq. (13).

graining volume of linear size $\ell=9$ lattice spacings for $d$ $=3$ and a square volume with $\ell=20$ for $d=2$. For $d=3$, given the very large size of the system we used only one run (there is no statistic average).

Let us first discuss the main qualitative features of the evolution of the spin configuration before entering the quantitative analysis of the time-dependent observables. Initial configurations are random, in that each spin points up or down with probability one half, which mimics an infinitely rapid quench from $T=\infty$. Initially, $\approx 66 \%$ for $d=3$ (56\% for $d=2$ ) sites satisfy the facilitation constraint and are able to flip with the heat bath probability weight. At the early stages of the evolution it remains relatively easy to find spins that satisfy the facilitation constraint. As time evolves the number of positive spins, and hence the magnetization, increases. However, as time passes one expects to have isolated spins and small islands of spins that point downwards. These may be remnants of the initial configuration or they may be due to "equilibrium-like" thermal fluctuations. The former are blocked until other down-pointing spins diffuse. The interior of the latter can flip but their border might be blocked due to the facilitated rule. A crossover (in the total time) between the "easy" initial regime and the "hard" latter regime was predicted in Ref. 43.

The analysis of the evolution for $d=3$ towards its equilibrium value gives a first indication of the apparent "glass transition" temperature. Figure 1 shows the time dependence of the magnetization density at $T=0.4$ on a log-linear scale. We see the above mentioned crossover occurring at $t_{\mathrm{CO}}$ $\sim 10^{3}$ MCS. The value of the magnetization at the longest time reached in the simulation $t=10^{8}$ MCS is still $10 \%$ away from the equilibrium value, $m_{\mathrm{EQ}}(T=0.4) \simeq 0.848$, showing that the system is still far from equilibrium. Assuming that the curve found for this range of times does indeed approach the equilibrium value and no further crossover to a different dynamic law occurs (a hypothesis that might not hold, see Ref. 52 for a more detailed analysis) we find that the second slower regime is well characterized by the law

$$
\begin{aligned}
& m(t) \sim m_{\mathrm{EQ}}(T=0.4)-A \log _{10}^{-a} t, \\
& m_{\mathrm{EQ}}(T=0.4) \simeq 0.848, \quad A=1.37, \quad a=1.22,
\end{aligned}
$$

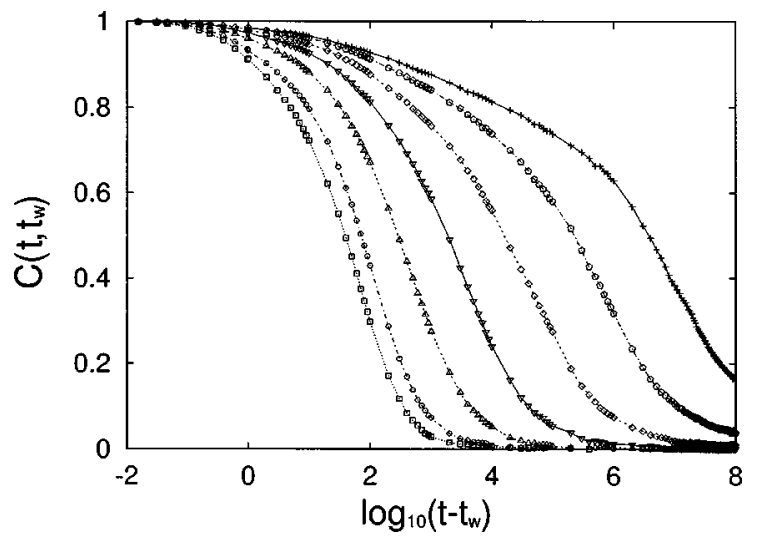

FIG. 2. The global two-time normalized connected correlation function $C\left(t, t_{w}\right)$ as a function of the logarithm of the time difference $t-t_{w}$, after a sudden quench to $T=0.4$. The curves correspond, from left to right, to seven logarithmically spaced values of the waiting-time $t_{w}=10^{1}, 10^{2}, 10^{3}, 10^{4}$, $10^{5}, 10^{6}, 10^{7}$ time steps. $d=3, L=128$.

demonstrating very slow dynamics even in the behavior of this one-time quantity. At higher temperatures one finds that the maximum magnetization reached in the numerical time window gets closer to the equilibrium value and, for example at $T \sim 0.7$ it actually reaches this value in $t \sim 10^{7}$ time steps. ${ }^{52}$ This value is considerably lower than the putative glass transition identified by Graham, Piché, and Grant. ${ }^{43}$ These authors might have overestimated this temperature by simulating very small systems (of maximum size $V=16^{3}$ ) as compared to the ones we use $\left(V=128^{3}\right)$.

What can we say about the structure of the remaining negative spins in the sample at long times? A set of snapshots of the instantaneous configuration shows that at around the crossover time there are no finite size domains left but only isolated spins pointing downward or, at most, very short strings of negative spins. It is then clear that their annihilation needs the cooperative diffusion of other spins in their neighborhood, which is a very slow process indeed.

The aging curves for the global connected and normalized correlation function defined in Eq. (13) are shown in Fig. 2 for several values of $t_{w}$ and as a function of $t-t_{w}$ in $\log$-linear scale. As discussed in Sec. II, disconnected correlation functions in glassy models usually show a two-step relaxation with a first rapid decay towards a plateau, that one estimates to be at $q_{\mathrm{EA}}(T) \sim m_{\mathrm{EQ}}^{2}(T)$ for a model undergoing simple domain growth, and a second slower aging decay towards zero. Connected correlation functions do not necessarily show a plateau. This is the case in domain growth. For the times explored numerically in the spin-facilitated model we do not see the development of the plateau (which is not even seen on a log-log scale). This might be due to the fact that the system is still very far from an asymptotic dynamic regime or it may simply mean that the connected correlation does not have a fast regime. ${ }^{52}$

We have attempted to scale the correlation data for all time differences and waiting times that are equal or longer than $t_{w}=10^{3}$ time steps (note that the curves for the two shorter waiting times are clearly different from the rest). We found that the scaling, 


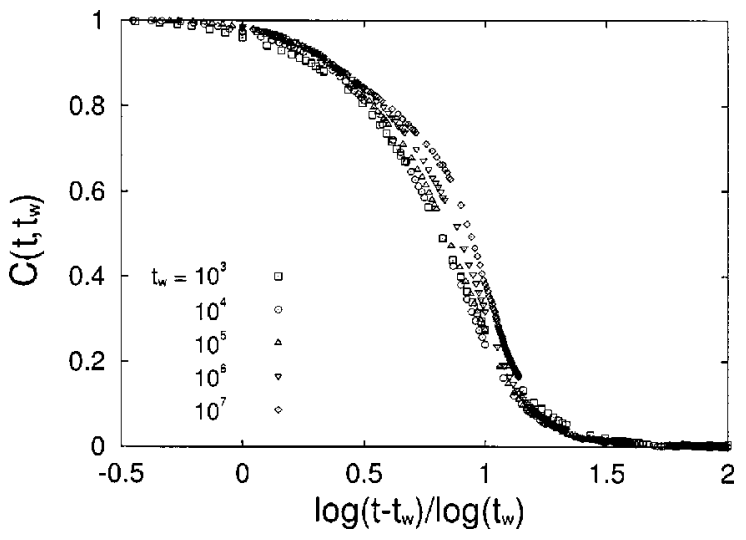

FIG. 3. Attempt to scale the global connected and normalized correlation function presented in Fig. 2. See the text for a discussion.

$$
C\left(t, t_{w}\right) \sim f\left(\frac{\ln \left(t-t_{w}\right)}{\ln t_{w}}\right)
$$

describes the data quite precisely for intermediate waiting times $\left(t_{w}=10^{2}-10^{4}\right.$ time steps), but fails to collapse the data for longer waiting times, as shown in Fig. 3. Interestingly, this scaling form is a precursor of dynamic ultrametricity. ${ }^{50,55}$ We have searched for other simple scaling forms without finding one that describe the full data in a satisfactory way. For this reason, we shall use the value of the global correlation itself to scale the PDFs of the local fluctuations. The same difficulty arises in the other kinetically constrained models that we have studied. Henceforth we omit the subin$\operatorname{dex} s$ that indicates the slow regime, since the kinetic facilitated models we have analyzed do not seem to have a first fast regime in their connected correlations.

Let us now turn to the study of the fluctuations of the local correlation functions $C_{r}^{\circ}\left(t, t_{w}\right)$ as defined in Eq. (10) and normalized to unity at equal times. What we clearly observe is that, even in this preasymptotic regime (in the sense that the magnetization, a one-time quantity, is far from its asymptotic value), the scaling of the PDF of local correlations is the same as the scaling of the global function. Moreover, the qualitative behavior of the form of the PDFs, their skewness and other momenta, are similar to the ones that Cipelletti et al. found experimentally. ${ }^{10}$ Working at different temperatures or using different linear sizes does not modify this conclusion.

The summary of the features shown by the PDFs of local connected functions is the following. Figure 4 shows a first indication of the evolution of the PDF of local connected functions (13) for fixed $t_{w}=10^{4}$ time steps and several values of $t-t_{w}$. Initially, the curves are asymmetric with respect to their average at short values of $t-t_{w}$, as exhibited by the curves for $t-t_{w}=1$ and 10 time steps. When $t-t_{w}$ increases they slowly approach a Gaussian normal form as shown by the curve for $t-t_{w}=1584$ time steps. Still later they go beyond the Gaussian form, and they skew in the opposite direction, e.g., $t-t_{w}=63095$ time steps. Finally, the curves very slowly tend to approach the Gaussian form again, e.g., $t-t_{w}=398107$ time steps.

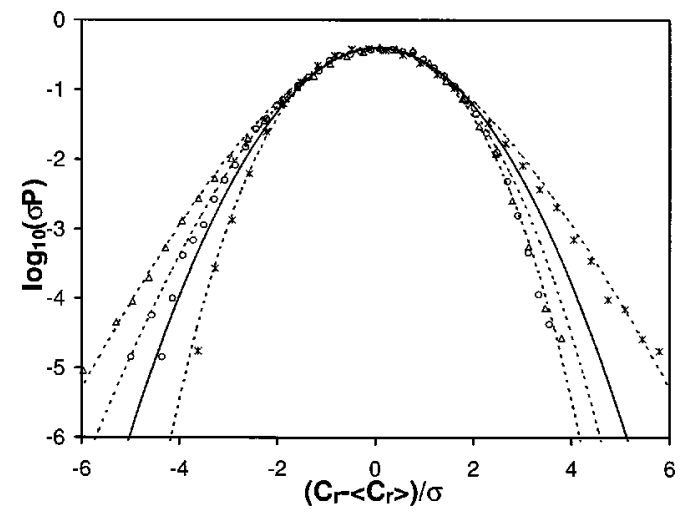

FIG. 4. Normalized PDF of the local correlations $C_{r}\left(t, t_{w}\right)$ in the modified FA model, $d=3, L=100, \ell=5$, and $T=0.4$. All curves drawn with symbols correspond to the waiting time $t_{w}=10^{4} \mathrm{MCS}$ at various the time differences $t-t_{w}$. The solid line represents the Gaussian normal form. The dashed lines are fits to modified Gumbel forms with parameter $a$ given by $a=13$ for $t$ $-t_{w}=1, a=50$ for $t=1600$, and $a=-13$ for $t=400000$.

The skewed PDFs resemble generalized Gumbel's distribution (see Appendix A). Even if this result is most probably just an approximation, and we cannot give it a probabilistic interpretation, it is worth mentioning the resemblance here. In Sec. VI we argue that these forms, and their evolution in time, can be understood using an effective random manifold theory that naturally gives rise to PDFs with a Gumbel-like form.

The next point we investigate is whether the PDFs of local correlations in the slow regime of widely separated times scale as the global correlation itself, namely,

$$
P\left(C_{r} ; t, t_{w}\right)=g\left[C_{r} ; C\left(t, t_{w}\right)\right] .
$$

In Refs. 20 and 21 we studied the $3 d$ EA model whose slow global correlation can be described with $h_{S}(t)=t$. Thus, we checked if

$$
\begin{aligned}
P\left(C_{r} ; t, t_{w}\right) & =g\left[C_{r} ; C_{S}\left(t, t_{w}\right)\right] \\
& =g\left[C_{r} ; f\left(\frac{h_{S}\left(t_{w}\right)}{h_{S}(t)}\right)\right] .
\end{aligned}
$$

Since we have not found a simple scaling form for global correlations in the kinetically constrained models, we test the hypothesis (15) directly. To this end, we chose pairs of total and waiting times $t$ and $t_{w}$ such that the global correlation takes the same value, say $\mathcal{C}$,

$$
C\left(t^{(k)}, t_{w}^{(k)}\right)=\mathcal{C} \quad \text { for } k=1, \ldots, n
$$

and we plot the PDFs of local correlations

$$
P\left[C_{r}\left(t^{(k)}, t_{w}^{(k)}\right)\right] \text { for } k=1, \ldots, n .
$$

The hypothesis (15) implies that for fixed value of $\mathcal{C}$ these PDFs should collapse onto a master curve.

In Fig. 5 we show the rather good demonstration of master collapse onto the Gumbel form for the modified FA model. To demonstrate that the strongly skewed distributions that we observe arise from nontrivial dynamics induced by facilatation, we construct a toy model that contains the same lattice features as the FA model but evolves via unconstrained dynamics. This model is discussed in detail in Ap- 

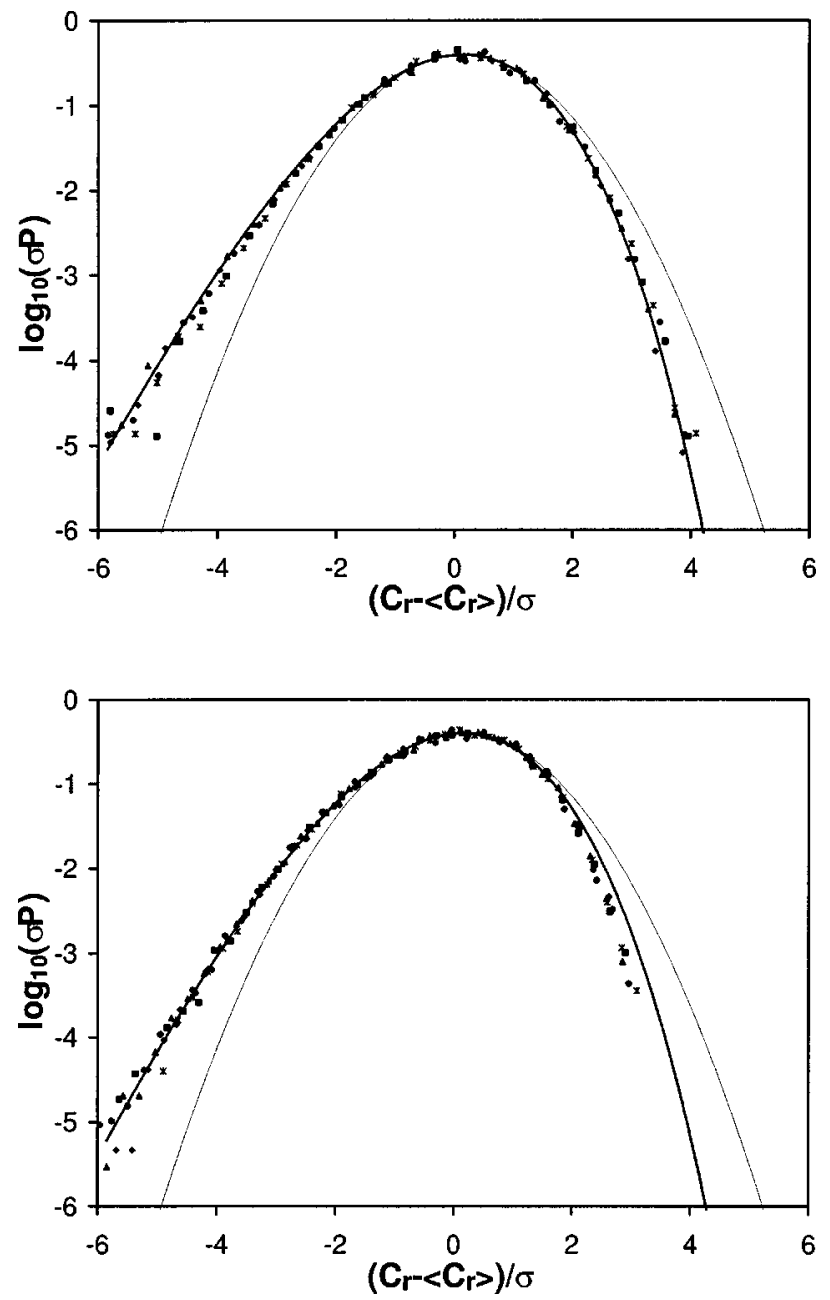

FIG. 5. Scaling of the normalized PDF of local correlations in the modified FA model, $d=3, L=100, \ell=5$, and $T=0.4$. $\mathcal{C}=0.88$ (top) and $\mathcal{C}=0.70$ (bottom). In both panels the data points correspond to five pairs of $t$ and $t_{w}$, with $t_{w}=10^{1}, \ldots, 10^{5}$ time steps. The thin line is a Gaussian normal form. The dark solid lines are fits to a Gumbel form with parameter $a=11$ (top) and $a=13$ (bottom).

pendix B. As shown in Fig. 6, the distribution of local correlations in this model is much weaker, and may be completely attributed to binomial-like fluctuations due to small coarse-grained cells. Furthermore, the data show no regime of approximate collapse onto a master curve, and for larger values of $t_{w}$ the distributions rapidly evolve to a Gaussian form. The nontrivial form and approximate scaling of the PDF of local correlations results from the dynamical constraints that induce a nontrivial dynamic length scale.

Now we look at the $d=2, p=2$ version of the model. In Fig. 7, we show the behavior of the normalized two-time connected correlation functions. As in the case of $d=3 p$ $=3$, we could find no global scaling of the decay for all waiting times. Figure 7 also shows the collapse of local correlations with $\mathcal{C} \sim 0.7$ onto the Gumbel form. Interestingly, while the Gumbel parameters may depend sensitively on temperature, dimensionality and the value of $\mathcal{C}$ for which the collapse is plotted, the scaling onto the Gumbel form is remarkably accurate.
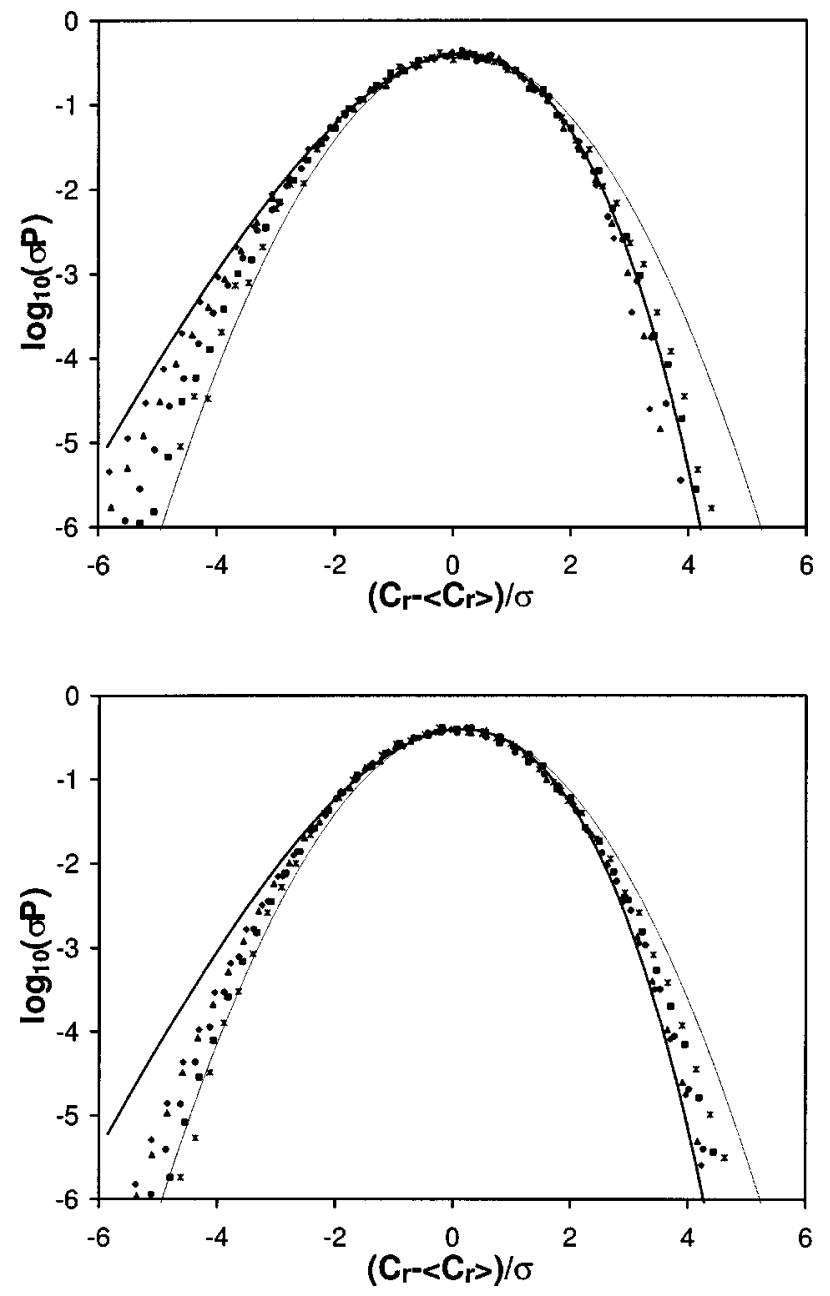

FIG. 6. Scaling of the normalized PDF of local correlations calculated from the toy model for the parameters corresponding to the conditions described in Fig. 5. Notice that the lack of data collapse in the dynamics toy model shows that the collapse we find in Fig. 5 is a consequence of a nontrivial correlated dynamics.

\section{Edwards-Anderson disordered spin system}

Similar results have been obtained for the disordered EA spin-glass model with usual Monte Carlo dynamics. In Fig. 8 we show the PDFs of global correlations and integrated responses for a small system. In this case, since the system is not macroscopic, we explore "mesoscopic" fluctuations that include similar information as the local fluctuations when $L$ is not much larger than the correlation length. We see that the generic behavior of the data is very similar to the one displayed in the previous section, although the particular time scaling [of the form (4) and (5) in this case] is different. The data is also described for time differences that are shorter than the characteristic relaxation time by a negatively skewed form that resembles a modified Gumbel distribution. The trend in $t-t_{w}$ is the same one although the approach to the Gaussian form is much slower and this asymptotic form is hardly reached within the time window explored. We have also simulated the procedure used by Cipelletti et al., i.e., we have measured temporal fluctuations in mesoscopic samples, and found very similar results.

It should be emphasized again that the glassy physics 

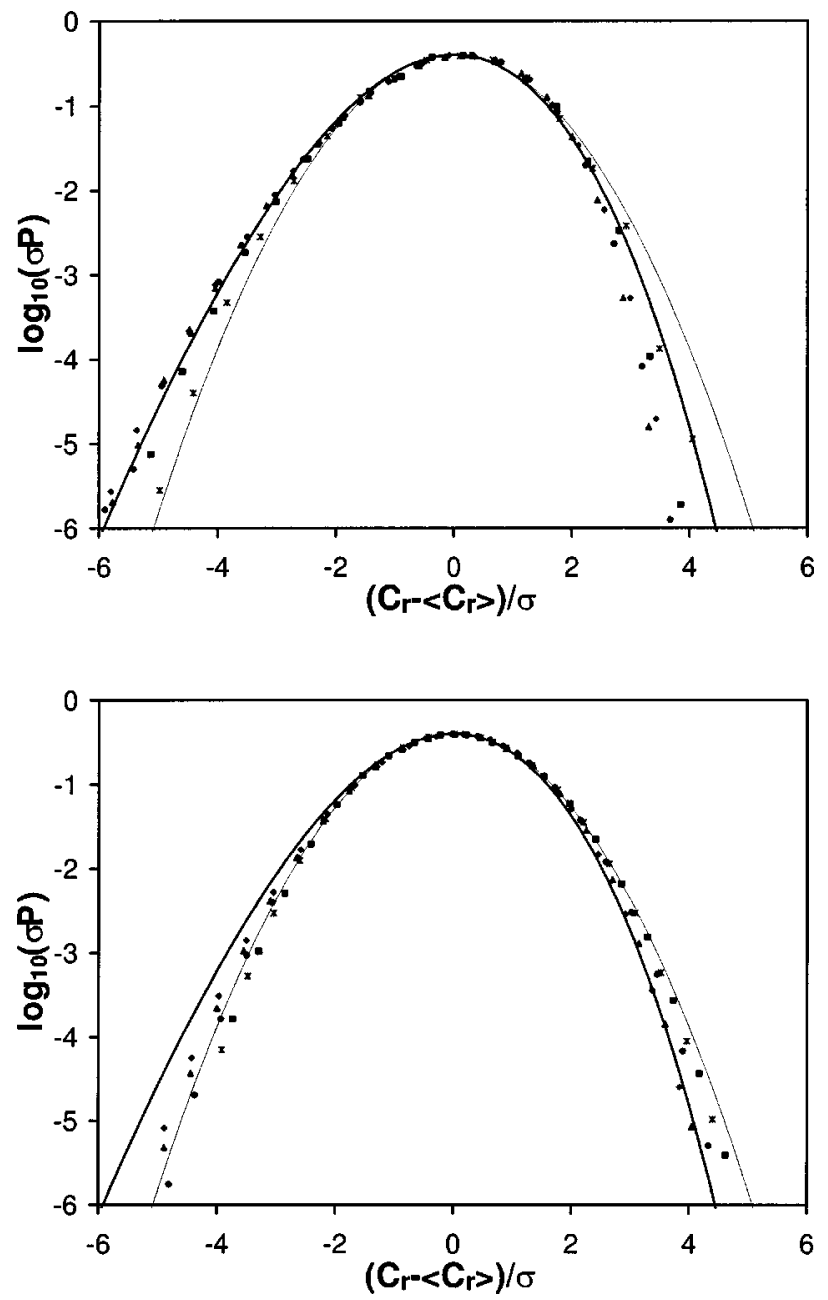

FIG. 7. Correlations in the modified FA model, $d=2$ and $L=1000$. Top: Scaling of the PDFs with $\mathcal{C} \sim 0.7$ at the same temperature for mesoscopic blocks of size $\ell=10$. The data correspond to five pairs of $t$ and $t_{w}$, with $t_{w}=10^{1}, \ldots, 10^{5}$ time steps. The dotted line is the Gaussian normal form. The dark solid line is a fit to a Gumbel form with $a=35$. Bottom: Toy model results for conditions corresponding to the top panel.

behind the EA model and the FA model differs in that a spin-glass transition exist at finite temperatures in the EA model, while no thermodynamic transition exists at finite temperature in the FA model. The growing correlation length in the FA model is of a dynamical nature. We have verified that the Gumbel-like distributions systematically revert to Gaussian distributions when the cell size is made larger. This suggests a direct way to estimate dynamical correlation lengths. Work along these lines is underway and will be reported in a future publication.

\section{ANALYTIC ARGUMENTS}

In this section we recall and extend some of the theoretical arguments introduced in Refs. 20, 21, and 35 to describe local and mesoscopic fluctuations in glassy systems. In those papers we focused on the time scaling of the PDFs without paying special attention to the form of these distributions. Here, instead, we investigate their form and we develop an effective theory to describe them.
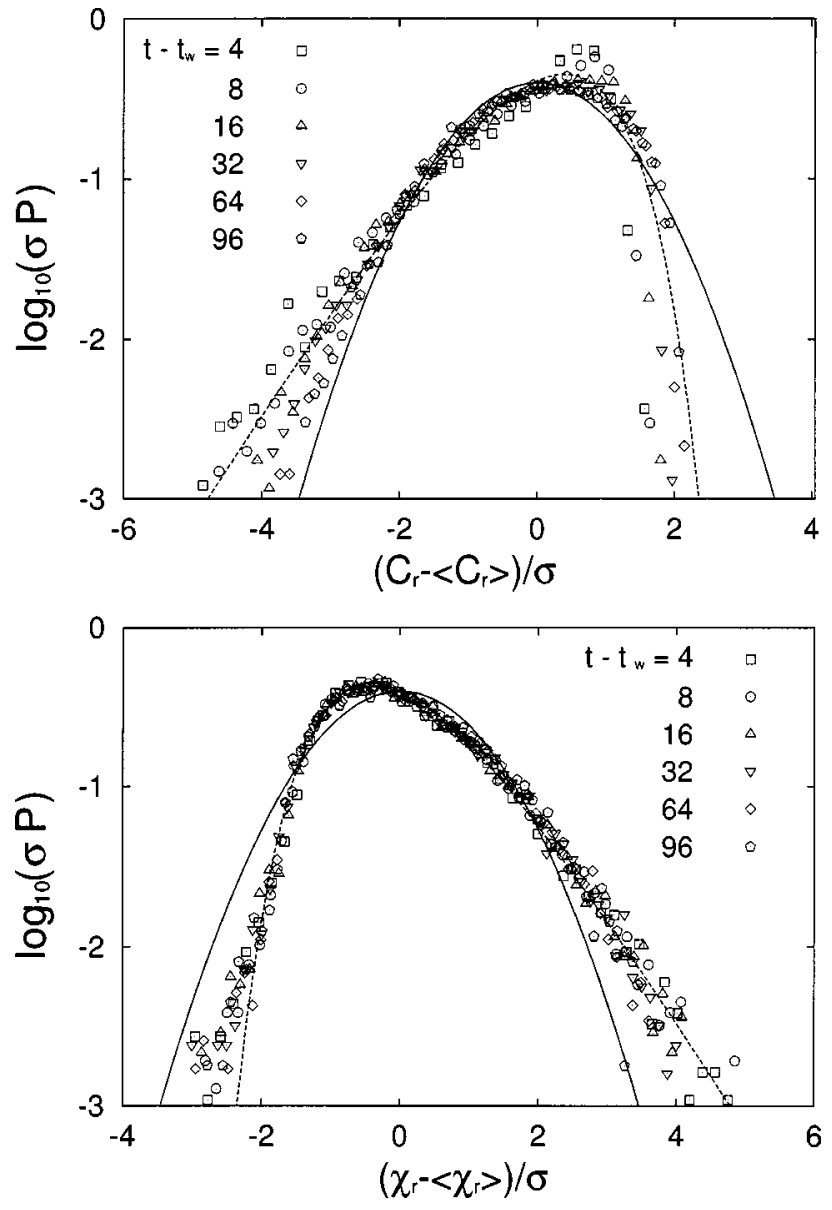

FIG. 8. Normalized PDFs of global correlation $C_{r}$ and integrated response $\chi_{r}$ (as defined in Ref. 2) for a small $3 d$ EA system with linear size $L=8$, temperature $T=0.4$ and waiting time $t_{w}=1000$. Data averaged over 8000 samples. The full line is the Gaussian distribution, while the dashed line is the generalized Gumbel form with $a=\pi / 2$.

\section{A. Reparametrization invariance}

The two-time scaling of correlation functions that are monotonic functions of the two times take the form

$$
C\left(t, t_{w}\right) \sim f\left(\frac{k\left(t_{w}\right)}{k(t)}\right)
$$

within each "correlation scale" as defined in Ref. 50. In Sec. $\mathrm{V}$ we discussed the dynamic evolution of three kinetically constrained models and the $3 d$ EA spin glass. For the latter, the aging decay is described quite well by a two-scale relaxation, see Eqs. (4) and (5), with a "simple aging" form, $h_{S}(t)=t$, for the slow part. For the kinetically constrained models we have not found a single function $h_{S}(t)$ that describes the full slow decay from $\mathcal{C}=1$ to $\mathcal{C}=0$ for all waiting times and times explored.

In order to keep the presentation as simple as possible, we discuss the theoretical arguments that allow one to predict the behavior of local fluctuations, assuming that the full slow decay is represented by the scaling given in Eq. (19) with a single function $k(t)=h_{S}(t)$. By the end of this section we argue how this approach can be extended to describe the case of a more complicated slow relaxation that does not fall 
into this category. (The failure may be due to the development of many correlation scales in the slow relaxation.)

In Ref. 35 it has been shown that the dynamic action controlling the local coarse grained dynamics at long waiting time $t_{w}$ and long time differences $t-t_{w}$ in the EA model approaches a form that is invariant under global reparametrizations of time that act as

$$
t \rightarrow h(t),
$$

with $h(t)$ a monotonically growing function. This change transforms the "fields" in this action in a special way that, at the level of expectation values, corresponds to leaving invariant the local correlations, $C_{r}\left(t, t_{w}\right) \rightarrow C_{r}\left[h(t), h\left(t_{w}\right)\right]$, and the integrated linear responses, $\chi_{r}\left(t, t_{w}\right)$ $\rightarrow \chi_{r}\left[h(t), h\left(t_{w}\right)\right]$. Symmetry breaking terms exist but they become smaller and smaller (irrelevant) as times and time differences increase. These symmetry breaking terms (which have their origin in the details of the short-time and shorttime-difference dynamics), although scaling down to zero at long times, are responsible for selecting a particular parametrization for which the global $\mathcal{C}$ decays from $q_{\mathrm{EA}}$ to 0 as $t$ is increased. Notice that if reparametrization invariance is left unbroken, $\mathcal{C}$ must be a constant independent of the times. Without entering the details of this action, let us introduce the main consequences of the existence of this approximate invariance.

Due to the global invariance, and the scaling in Eq. (19), one can argue that the slow part of the coarse-grained local correlations scale in time as Refs. 20, 21, and 35:

$$
C_{r}^{s}\left(t, t_{w}\right) \sim f\left(\frac{h_{r}\left(t_{w}\right)}{h_{r}(t)}\right),
$$

where the functions $h_{r}(t)$ are local time reparametrizations, $t \rightarrow h_{r}(t)$. The superscript $s$ means that we are looking here at the slow part of the decay of the local correlation only (in other words, the decay for long time differences compared to the waiting time $t_{w}$ ). The argument in Eq. (21) varies from unity when the times tend to be equal, $t \rightarrow t_{w}^{+}$, to zero at widely separated times $t \gg t_{w}$. The external function $f$ takes values between $f(1)=q_{\mathrm{EA}}$ and $f(0)=0$. The first limit is independent of the position $r$ since one does not expect the plateau to be a fluctuating quantity if a sufficiently large coarse-graining volume is used. The second limit holds if there is no external field applied and all local correlations decay to zero. We consider here monotonic local reparametrizations of the times, such that $h_{r}\left(t_{w}\right)<h_{r}(t)$ at all $r$ for $t_{w}<t$. This is equivalent to assuming that the time lags we shall consider are not extremely short.

Our original claim was that the external function $f$ is the same for all coarse-grained centers in the sample, while all spatial fluctuations are encoded in the internal function $h_{r}$. The reason for this proposal is that the global reparametrization invariance in time of the dynamic action in this two-time regime leads to low action excitations (Goldstone modes) for smoothly varying spatial fluctuations in the reparametrization of time, but not in the external form of the correlations. As in a sigma model, the external function $f$ fixes the manifold of states, and the local time reparametrizations corre- spond to fluctuations restricted to this fixed manifold of states. The relation to the $\sigma$ model is described in detail in Ref. 21 and we shall not repeat it here.

The symmetry-based argument and its predictions, originally developed on the basis of the time-reparametrization invariance of the dynamical action of the EA model, was proposed to hold beyond this model whenever a separation between fast and slow decay in the global correlation and response develops in time. Our results on models without quenched disorder conclusively demonstrate the generality beyond the EA model.

\section{B. Effective random manifold action}

As in the sigma model approach to an interacting system in which we have identified a relevant variable and a relevant symmetry, we propose an effective action for the relevant degree of freedom that quantifies the fluctuations about the globally symmetric result. The philosophy is the same as the one followed when describing spin-wave excitations in a Heisenberg ferromagnet with a quadratic action that depends on the angular variables only.

The scaling form in Eq. (21) can be written in an equivalent form by defining $\phi_{r}(t) \equiv \ln h_{r}(t)$ :

$$
\begin{aligned}
C_{r}^{S}\left(t, t_{w}\right) & \sim f\left(e^{-\left[\phi_{r}(t)-\phi_{r}\left(t_{w}\right)\right]}\right) \\
& =f\left(e^{-\int_{t_{w}}^{t} d t^{\prime} \phi_{r}\left(t^{\prime}\right)}\right) \equiv f\left(e^{-\Delta \phi_{r} t_{t_{w}}^{t}}\right) .
\end{aligned}
$$

In the dynamic case under study, after parametrizing the correlation in this way, the relevant field is the function $\phi_{r}(t)$. The action governing the dynamics of $\phi_{r}(t)$ depends on the details of the particular problem. However, we can greatly restrict the form of the possible actions by simply using the constraints due to the symmetries. These are:

(i) The action must be invariant under a global time reparametrization $t \rightarrow s(t)$.

(ii) If our interest is in short-ranged problems, the action must be written using local terms. The action can thus contain products evaluated at a single time and point in space of terms such as $\phi_{r}(t), \dot{\phi}_{r}(t), \nabla \phi_{r}(t), \nabla \dot{\phi}_{r}(t)$, and similar derivatives.

(iii) The scaling form in Eq. (22) is invariant under $\phi_{r}(t) \rightarrow \phi_{r}(t)+\Phi_{r}$, with $\Phi_{r}$ independent of time. Thus, the action must also contain this symmetry.

(iv) The action must be positive definite.

These requirements largely restrict the possible actions. The action with the smallest number of spatial derivatives (most relevant terms) is

$$
S_{\mathrm{EFF}}=\int d^{d} r \int d t\left[K \frac{\left[\nabla \dot{\phi}_{r}(t)\right]^{2}}{\dot{\phi}_{r}(t)}+M \dot{\phi}_{r}(t)\right] .
$$

The last term is simply a total derivative, and its space-time integral is constant, so we shall drop it. Hence, we find that the strong constraints imposed on the action reduces it to the form

$$
S_{\mathrm{EFF}}=K \int d^{d} r \int d t \frac{\left[\nabla \dot{\phi}_{r}(t)\right]^{2}}{\dot{\phi}_{r}(t)} .
$$


Notice that the action now solely depends on the time derivatives $\dot{\phi}_{r}(t)$.

Let us now examine the consequences of this action on the form of the local correlations (22). Due to the simple form (24) the $\dot{\phi}_{r}$ are uncorrelated at any two different times $t_{1}$ and $t_{2}$. Thus the expression $\left.\Delta \phi_{r}\right|_{t_{w}} ^{t}=\int_{t_{w}}^{t} d t^{\prime} \dot{\phi}_{r}\left(t^{\prime}\right)$ entering the exponential in the scaling form in Eq. (22) is a sum of uncorrelated random variables in time. Hence, one can interpret such expression as the displacement of a random walker with position dependent velocities. Alternatively, one can think of the space-dependent differences $\left.\Delta \phi_{r}\right|_{t_{w}} ^{t}$ $=\int_{t_{w}}^{t} d t^{\prime} \dot{\phi}_{r}\left(t^{\prime}\right)$ as the net space-dependent height (labeled by $t$ ) of a stack of spatially fluctuating layers $d t^{\prime} \dot{\phi}_{r}\left(t^{\prime}\right)$. The action for the fluctuating surfaces of each layer is given by Eq. (24).

Before proceeding, let us take a moment to show that indeed the action in Eq. (24) satisfies all the four constraints enumerated above. First, consider a global change of variables $t \rightarrow s(t)$, for which the action transforms as

$$
\begin{aligned}
S_{\mathrm{EFF}} \rightarrow K \int d^{d} r \int d t \frac{\left(\frac{d s}{d t}\right)^{2}\left(\nabla \dot{\phi}_{r}[s(t)]\right)^{2}}{\left(\frac{d s}{d t}\right) \dot{\phi}_{r}[s(t)]} \\
=K \int d^{d} r \int d s \frac{\left[\nabla \dot{\phi}_{r}(s)\right]^{2}}{\dot{\phi}_{r}(s)}=S_{\mathrm{EFF}} .
\end{aligned}
$$

Hence, as we required in point (i), the action is invariant under the global time reparametrization. The action is also clearly local. Because it only contains explicitly $\dot{\phi}_{r}$ but not $\phi_{r}$, it is invariant under $\phi_{r}(t) \rightarrow \phi_{r}(t)+\boldsymbol{\Phi}_{r}$, with $\boldsymbol{\Phi}_{r}$ independent of time. Finally, since the local reparametrizations $h_{r}(t)$ are monotonically increasing functions of time, so are $\phi_{r}(t)=\ln h_{r}(t)$, and consequently $\dot{\phi}_{r}(t)>0$. Hence, the combination $\left(\nabla \dot{\phi}_{r}\right)^{2} / \dot{\phi}_{r}$ is always positive, and the action is positive definite.

To better understand the consequences of this effective action, we study it in two regimes. The first is one in which we consider only small spatial fluctuations of the local reparametrizations with low velocities. In the second, we consider the full effect of the action, without assuming that the local fluctuations are small. We will use the latter to obtain the full form of the probability distribution of local correlations and compare it to the numerical and experimental results.

\section{Linear regime}

We begin by making some simplifying assumptions so as to obtain a better understanding of the consequences of local reparametrizations. ${ }^{20,35}$ We shall shortly relax these assumptions. If we assume that the site-to-site fluctuations are small, the field $\phi_{r}(t)$ will be given by

$$
\phi_{r}(t)=s(t)+\varphi_{r}(t) \quad \text { with } \varphi_{r}(t) \ll s(t),
$$

and the uniform part $s(t)$ defined as

$$
s(t) \equiv \ln h_{S}(t)
$$

with $h_{S}(t)$ the scaling function of the global correlation. In terms of the $s$ parametrization we can write

$$
\phi_{r}(s)=s+\varphi_{r}(s)
$$

and

$$
\dot{\phi}_{r}(s)=1+\dot{\varphi}_{r}(s) .
$$

We shall further assume that

$$
\dot{\varphi}_{r}(s) \ll 1 \text {. }
$$

This approximation leads to the action

$$
S_{\mathrm{EFF}} \approx K \int d^{d} r \int d s\left[\nabla \dot{\varphi}_{r}(s)\right]^{2}
$$

The surfaces $\dot{\varphi}_{r}(s)$ are uncorrelated for different reparametrized times $s$. The argument that enters in the evaluation of the local correlation functions $C_{r}\left(t, t_{w}\right)$ is, according to Eq. (22),

$$
\begin{aligned}
\left.\Delta \phi_{r}\right|_{t_{w}} ^{t} & =\int_{s\left(t_{w}\right)}^{s(t)} d s\left[1+\dot{\varphi}_{r}(s)\right] \\
& =s(t)-s\left(t_{w}\right)+\sqrt{s(t)-s\left(t_{w}\right)} X_{r} \\
& =\ln \frac{h_{S}(t)}{h_{S}\left(t_{w}\right)}+\sqrt{\ln \frac{h_{S}(t)}{h_{S}\left(t_{w}\right)}} X_{r},
\end{aligned}
$$

where the square root arises from the fact that the $\dot{\varphi}$ surfaces are uncorrelated for different $s$, leading to a sum of independent variables in the reparametrized time direction. The $X_{r}$ is a Gaussian random surface that encodes the spatial fluctuations, and it is distributed with probability

$$
P\left(X_{r}\right) \propto e^{-K \int d^{d} r\left(\nabla X_{r}\right)^{2}} .
$$

Even within the linear approximation (29) and (30), one can already explain why the probability distributions for local correlations of a system with a global scaling as in (4) and (5) collapse as a function of $h_{S}(t) / h_{S}\left(t_{w}\right)$. This fact is a consequence of Eqs. (22) and (32). ${ }^{20,21}$ Moreover, noticing that the two-time global correlation is in one-to-one correspondence with the ratio $h_{S}(t) / h_{S}\left(t_{w}\right)$, one concludes that the PDF of local correlations scales in time as the global correlation itself.

While this approximation gives the observed time scaling of the PDFs of local correlators, it does not give their correct shape, in particular when $t \rightarrow t_{w}^{+}$. The reason is that the assumption that site-to-site fluctuations and their velocities are small as compared to the bulk average fails more severely in this regime. As we show next, we can still tackle the problem in the nonlinear regime.

\section{Nonapproximate treatment of fluctuations}

In order to get a more accurate prediction of the form of the PDF, one needs to go beyond the approximations in Eqs. (26) and (30). As done in the previous section, we start by writing the action in the parametrization $s(t)=\ln h_{S}(t)$ chosen by the bulk values, and by defining a new field ${ }^{59} \psi_{r}(s)$ such that $\psi_{r}^{2}(s)=\dot{\phi}_{r}(s)$, one then has 


$$
S_{\mathrm{EFF}}=K \int d^{d} r \int d s\left[\nabla \psi_{r}(s)\right]^{2} .
$$

This is again the action of uncorrelated Gaussian surfaces for different proper times $s$.

The argument entering in Eq. (22) now reads

$$
\left.\Delta \phi_{r}\right|_{t_{w}} ^{t}=\int_{s\left(t_{w}\right)}^{s(t)} d s \dot{\phi}_{r}(s)=\int_{s\left(t_{w}\right)}^{s(t)} d s \psi_{r}^{2}(s),
$$

Due to the Gaussian statistics of the $\psi_{r}(s)$, it is simple to show that connected $N$-point correlations of $\left.\Delta \phi_{r_{1}}\right|_{t_{w}} ^{t}$ satisfy

$$
\begin{aligned}
& \left\langle\left.\Delta \phi_{r_{1}}||_{t_{w}}^{t} \Delta \phi_{r_{2}}\right|_{t_{w}} ^{t} \cdots \Delta \phi_{r_{N}} \mid t_{w}\right\rangle_{c} \\
& =\left[s(t)-s\left(t_{w}\right)\right] \mathcal{F}\left(r_{1}, r_{2}, \ldots, r_{N}\right),
\end{aligned}
$$

where the function $\mathcal{F}$ can be obtained from Wick's theorem, summing over all graphs that visit all sites (connected) with two lines (because of $\psi^{2}$ ) for each vertex $i$ corresponding to a position $r_{i}$. Notice that the reparametrized times appear only in the prefactor $\Delta s=s(t)-s\left(t_{w}\right)$. Therefore, time dependencies are functions of $\Delta s$ alone. Once again we find that the probabilistic features of the fluctuations of local correlations are functions of the scaling variable, $\Delta s$ $=\ln h_{S}(t) / h_{S}\left(t_{w}\right)$, and hence of the global correlation itself. This coincides with the numerical observations.

Here, a comment is in order. In obtaining the effective action, we have neglected higher gradients and time derivatives because these were considered irrelevant. In particular, this is justified for large reparametrized time differences. Note, however, that these terms are important when $\Delta s$ becomes of order unity. This short (reparametrized) time-cutoff scale means that the surfaces for different $s_{1,2}$ are uncorrelated only when $\left|s_{1}-s_{2}\right|$ is larger than a cutoff $\delta s$. Keeping this in mind, let

$$
\left.\Delta \phi_{r}\right|_{t_{w}} ^{t}=\delta s \sum_{i=1}^{n} \psi_{r}^{2}\left(s_{i}\right),
$$

where we have discretized the integral in Eq. (37) in steps of the cutoff $\delta s$. The number of such steps is $n=\Delta s / \delta s$. The expression (35) and the scaling relation (22) allow us to compute the PDF for the local correlation functions [these expressions can also be used to investigate $\mathrm{N}$-point correlations of $\left.C_{r}\left(t, t_{w}\right)\right]$. We shall do this in the following section generating numerically random Gaussian surfaces $\psi_{r}(s)$, from which we obtain $\left.\Delta \phi_{r}\right|_{t_{w}} ^{t}$ and then $C_{r}\left(t, t_{w}\right)$. Let us now discuss the extreme cases (when $n$ is either small or large) before turning to the numerical calculations, which are better suited to describe the crossover between these two limits.

Case $\mathcal{C} \approx q_{\mathrm{EA}}$. If the global correlation is close to the Edwards-Anderson parameter, $n=\Delta s / \delta s$ is small. Consider for simplicity $n=1$. In this case, letting $Y_{r}=\psi_{r}\left(s_{1}\right)$, we write

$$
\left.\Delta \phi_{r}\right|_{t_{w}} ^{t}=\delta s Y_{r}^{2},
$$

with $Y_{r}$ distributed according to

$$
P\left(Y_{r}\right) \propto e^{-K \int d^{d} r\left(\nabla Y_{r}\right)^{2}} .
$$

Using Eq. (22) the local correlation is given by

$$
C_{r}\left(t, t_{w}\right)=\int\left(e^{-\left.\Delta \phi_{r}\right|_{t_{w}} ^{t}}\right)=\int\left(e^{-\delta s Y_{r}^{2}}\right) .
$$

In what follows, we connect the PDFs of the local correlations $C_{r}$ coarse grained over a volume around the point $\mathbf{r}$ and the PDFs for the fluctuations of the global magnetization $m$ of the $X Y$ magnet at low temperature, as studied by Bramwell et al. ${ }^{38}$ or the roughness of a Gaussian surface of the Edwards-Wilkinson type, as analyzed by Rácz and co-workers. ${ }^{37}$ For concreteness, we use in the following the language of the $X Y$ model.

In the case of the $X Y$ magnet, the local field $\theta_{r}$ is distributed according to

$$
P\left(\theta_{r}\right) \propto e^{-K \int d^{d} r\left(\nabla \theta_{r}\right)^{2}},
$$

and one can write the local magnetization in one direction as

$$
m_{r}=\cos \theta_{r},
$$

where for simplicity we took $m=1$. Bramwell et al. found that the distribution of the global magnetization of finite-size systems does approach a scale-invariant non-Gaussian distribution (when the magnetization is normalized by subtracting the average and dividing by the standard deviation). In particular, the PDF is skewed and can be approximated by a generalized Gumbel distribution. The underlying reason for the nonapplicability of the central limit theorem is that the system is critical (there is no finite correlation length) and hence the local magnetizations are not uncorrelated. Similarly, in a system where the correlation length $\xi$ is finite, non-Gaussian distributions will be found as long as the coarse graining length is smaller than $\xi$.

The connection between the PDFs $P\left(C_{r}\right)$ and $P(m)$ for dynamic local coarse grained correlations $C_{r}\left(t, t_{w}\right)$ in our case and the global magnetization $m$ in the $X Y$ model is as follows. First, the distribution of the variables $Y_{r}$ and $\theta_{r}$ in Eqs. (39) and (41) are identical. The fact that the functional dependencies of $C_{r}$ and $m_{r}$ on $Y_{r}$ and $\theta_{r}$ respectively, as given in Eqs. (40) and (42), are not the same lead to slightly different forms of the PDFs that, in both cases, can be approximated by generalized Gumbel forms with different parameters. To illustrate this point, consider the case in which the stiffness $K$ is large so that both variables $Y_{r}$ and $\theta_{r}$ (centered around zero) are small within a given coarse graining volume. In this case, we can expand Eqs. (40) and (42) as follows:

$$
\begin{aligned}
& C_{r}=f\left(e^{-\delta s Y_{r}^{2}}\right) \approx f(1)-\delta s Y_{r}^{2} f^{\prime}(1)+\cdots \\
& m_{r}=\cos \theta_{r} \approx 1-\frac{1}{2} \theta_{r}^{2}+\cdots
\end{aligned}
$$

and because the PDFs are calculated for normalized variables (zero average and unit variance), the constants in the problem [such as $f(1)$ and $\int_{s}^{\prime}(1)$ ] have no effect on the distributions. Hence, in this case the PDFs for the two problems are identical. The sum over a finite volume, used to compute the global mesoscopic $m$ does not modify this conclusion. In particular, in $2 d$ the PDF can be approximated by a generalized Gumbel distribution with parameter $a \approx \pi / 2$ (see Appen$\operatorname{dix}$ A), as discussed in detail in Ref. 38. 
We want to stress that the description of the PDFs with extreme statistics is, in general, just an approximation that correctly captures their main features, namely a single maximum, negative skewness that crosses over to a positive skewness, and an exponential or near exponential tail for small fluctuations below the mean. Moreover, $C_{r}$ is not $a$ priori an extremal quantity so there is no deep reason why it should be distributed following the extreme value statistics.

Case $\mathcal{C} \approx 0$. If the global correlation is close to zero, $n$ $=\Delta s / \delta s$ is large. In this case, the sum in Eq. (37) has many terms, and since all terms in the sum are positive, the total will have some average that is proportional to $\Delta s$ plus fluctuations. Some reflection point us to the same situation that we presented in Sec. VIB 1, where we discussed the linear regime. Thus, we can write the local correlations as

$$
C_{r}=f\left(e^{-\Delta s-\sqrt{\Delta s} X_{r}}\right),
$$

where the variable $X_{r}$ is distributed according to Eq. (33). From here one can show that if $f(x)$ is a monotonic increasing function, the PDF $P\left(C_{r}\right)$ is positively skewed (as opposed to what is found when $\left.\mathcal{C} \approx q_{\mathrm{EA}}\right)$. Indeed, expanding $C_{r}$ around $X_{r} \sim 0$,

$$
\begin{aligned}
C_{r} \approx & f\left(e^{-\Delta s}\right)-\sqrt{\Delta s} X_{r} e^{-\Delta s} f^{\prime}\left(e^{-\Delta s}\right) \\
& +\frac{\Delta s}{2} X_{r}^{2}\left[e^{-2 \Delta s} f^{\prime \prime}\left(e^{-\Delta s}\right)+e^{-\Delta s} f^{\prime}\left(e^{-\Delta s}\right)\right]
\end{aligned}
$$

one sees that the signs of the quadratic term in $X_{r}$ in Eq. (47) and the quadratic term in $Y_{r}$ in Eq. (43) have opposite signs. Since this sign dictates the skewness (recall that $X_{r}$ has a symmetric distribution), one concludes that the PDF is positively skewed in this limit, as also observed numerically and experimentally.

\section{Beyond a single scale}

The argument used above can be heuristically extended to deal with more general problems. Let us assume that global correlations evolve in a sequence of scales, each characterized by their own scaling function $h_{k}(t)$ and external function $f_{k}^{23,50,56}$

$$
C\left(t, t_{w}\right)=\sum_{k=1}^{n} f_{k}\left(\frac{h_{k}\left(t_{w}\right)}{h_{k}(t)}\right)
$$

with each $h_{k}(t)$ different monotonically growing function of the original time. This means that when the ratio for a chosen value of $k$ varies as

$$
0 \leqslant \frac{h_{k}\left(t_{w}\right)}{h_{k}(t)} \leqslant 1,
$$

all others are constant:

$$
\frac{h_{k^{\prime}(<k)}\left(t_{w}\right)}{h_{k^{\prime}(<k)}(t)}=0, \quad \frac{h_{k^{\prime}(>k)}\left(t_{w}\right)}{h_{k^{\prime}(>k)}(t)}=1 .
$$

The boundary conditions are such that

$$
\lim _{x \rightarrow 1^{+}} f_{k}(x)=q_{k}, \quad \lim _{x \rightarrow 0} f_{k}(x)=0,
$$

with $0 \leqslant q_{k+1}<q_{k} \leqslant 1$, and $\sum_{k=1}^{n} q_{k}=1$. In the time regime in which the $k$ th ratio varies the global correlation reads

$$
C\left(t, t_{w}\right)=f_{k}\left(\frac{h_{k}\left(t_{w}\right)}{h_{k}(t)}\right)+\sum_{k^{\prime}>k} q_{k^{\prime}} .
$$

Motivated by what was presented in $^{20,35}$ we propose that the local fluctuations can be parametrized as

$$
\begin{aligned}
C_{r}\left(t, t_{w}\right) & =\sum_{k=1}^{n} f_{k}\left(\frac{h_{k r}\left(t_{w}\right)}{h_{k r}(t)}\right) \\
& =\sum_{k=1}^{n} f_{k}\left(e^{\phi_{k r}\left(t_{w}\right)-\phi_{k r}(t)}\right) .
\end{aligned}
$$

We assume that the local fluctuations are small in the sense that they do not exchange scales. This is consistent with the supposition that the Edwards-Anderson parameter, or more generally in this case the plateau values $q_{k}$, are not modified by the fluctuations.

Based on the symmetry arguments already explained, we propose that each $\phi_{k_{r}}(t)$ is distributed as in (24). This leads, after the reparametrization of time, to

$$
s_{k}(t) \equiv \ln h_{k}(t),
$$

and the change of variables $\left(\psi_{k_{r}}\left(s_{k}\right)\right)^{2}=\dot{\phi}_{k_{r}}(t)$, to a Gaussian distribution for each $\psi_{k_{r}}\left(s_{k}\right)$,

$$
S_{\mathrm{EFF}}^{k}=K \int d^{d} r \int d s_{k}\left[\nabla \psi_{k_{r}}\left(s_{k}\right)\right]^{2} .
$$

Note that the $\dot{\phi}_{k_{r}}\left(s_{k}\right)$ are positive and we assumed that the stiffness $K$ is $k$ independent, for simplicity. This is simply due to the fact that the $h_{k}(t)$ characterizing each two-times sector are monotonically growing functions of time.

Thus, when we look at the connected correlations between the $\left.\Delta \phi_{k_{r}}\right|_{t_{w}} ^{t}$, for given values of $t$ and $t_{w}$ that correspond to the scale $k$ for the global correlation, only the $k$ th scale makes a nontrivial (nonconstant) contribution. Since in each two-time sector the global scaling of the PDF of local correlations is the same as the one for the global $\mathcal{C}$, we find that this result holds for all times. Once we have analyzed the case of $n$ scales, we can take the limit $n \rightarrow \infty$ that corresponds to the ultrametric scaling form and claim that in this case the PDFs scale as the global ones. Thus, we expect the collapse of the type found for the facilitated models.

\section{Numerics for random surfaces}

In this section we numerically generate random surfaces with the statistics given by Eq. (35) and we calculate the PDFs of local correlations related to the surfaces using Eq. (22). In order to carry out numerical evaluations of the local $C_{r}\left(t, t_{w}\right)$ PDFs let

$$
\left.\Delta \phi_{r}\right|_{t_{w}} ^{t}=\delta s \sum_{i=1}^{n} \psi_{r}^{2}\left(s_{i}\right),
$$

where we have discretized the integral in Eq. (35) in steps of the cutoff $\delta s$. We proceed as follows: 
(i) We generate independent Gaussian random surfaces $\psi_{r}\left(s_{i}\right)$, which we choose to be of unit variance since the choice of variance does not change the results qualitatively. These surfaces are generated with the distribution $P\left[\dot{\psi}_{r}\left(s_{i}\right)\right] \propto e^{-K \int d^{d} r\left(\nabla \dot{\psi}_{r}\right)^{2}}$ [see Eq. (34)].

(ii) We then sum the $\psi_{r}^{2}\left(s_{i}\right)$ over $n$ time slices. The number of slices determines the time difference $t-t_{w}$.

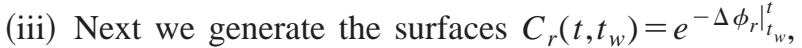
i.e., we choose for simplicity the "external" function $f(x)$ $=x$ [see Eq. (4)].

(iv) Finally, we look at the PDFs $P\left(C_{r}\right)$ of the normalized coarse-grained local correlations $C_{r}$ [subtracting the average, scaling by the standard deviation, and coarse-graining over a box of volume $\ell^{3}=(2 M+1)^{3}$ ] as a function of the number of slices $n$.

Before presenting the numerical results, let us discuss in more detail the issue of coarse graining the correlation $C_{r}$ over a certain volume. There are actually two levels of coarse graining for theories defined on a lattice. First, in order to define continuous fields $h_{r}(t), \phi_{r}(t)$, and ultimately $\psi_{r}(t)$, a coarse graining of a lattice theory has already occurred. Coarse-graining the local correlation $C_{r}$ over a given volume is a second level of coarse graining. The connection to the $X Y$ model and the fluctuations of the global magnetization studied by Bramwell et al. ${ }^{38}$ is helpful in clarifying this point. Starting from a lattice theory, one coarse grains to obtain a Landau-type theory of a continuous variable $m_{r}$. Then, to study the fluctuations of the total magnetization of the system over a given volume, one must average $m_{r}$ over a second coarse-graining volume (which in the case of the global magnetization is the entire volume of the system). In the numerical simulations we present here, we start from an action for the $\psi_{r}$ that is Gaussian. The first level of coarse graining that led to an action for a continuous $\phi_{r}$ or $\psi_{r}$ was assumed to have taken place already. So even though in the numerical calculations we employ a discretization, one should keep in mind that a first coarse-graining has been invoked.

In Fig. 9, we show for a system of size $L=32$ the dependence of the $P\left(C_{r}\right)$ on the number of slices. We show curves for $n=4,8,12,16,20$, and for a coarse-grained volume $\ell=3$. The smaller the value of $n$, the smaller is the ratio $h_{S}(t) / h_{S}\left(t_{w}\right)$. Several comments are in order. First, notice that for small $n$, the curves clearly deviate from a Gaussian form and are reasonably well fit by a generalized Gumbel distribution (see Appendix A). The curves become more Gaussian as $n$ increases. This behavior is qualitatively similar to the one we obtained for the facilitated spin model and the $3 d$ EA model we studied numerically in Sec. $\mathrm{V}$ as well as the experimental observations of Cipelletti et al. ${ }^{10}$ The approach to a Gaussian behavior is, however, very slow and we do not reach the Gaussian form with the values of $n$ used.

\section{E. Essential ingredients for universal fluctuations}

Why do the analytical arguments we presented above, based on a symmetry (reparametrization invariance), lead to the universal behavior and collapse (for a fixed global correlation) of the $P\left(C_{r}\right)$ that is observed in the facilitated spin
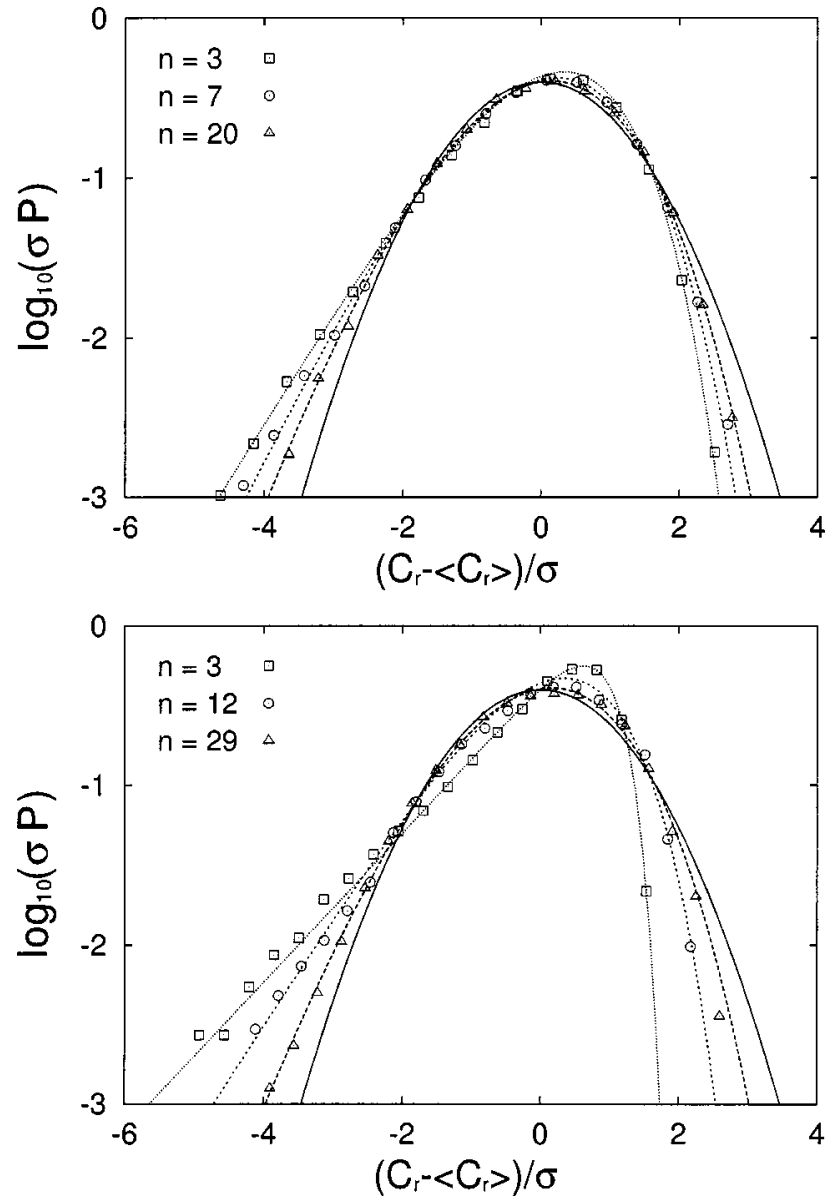

FIG. 9. Normalized PDFs $P\left(C_{r}\right)$ for local coarse-grained correlations $C_{r}$ generated by the random surfaces suggested by the analytical theory. The curves shown are for sums over $n$ slices. As $n$ increases they become less skewed and approach the Gaussian distribution (full line). Top: $d=3$, system of linear size $L=32$, and the coarse-graining cell of linear size $\ell=3$. The Gumbel parameter is $a=2.2,4.5,11$ (for increasing $n$ ). Bottom: $d=2, L$ $=32$, and $\ell=5 ; a=0.4,2,10$ (for increasing $n$ ).

models, in the $3 d$ Edwards-Anderson model, and in some experiments ? $^{10}$ We can single out two essential elements of the theoretical description that allow it to account for this behavior.

The first ingredient is that the theory proposed in Refs. 20, 21, and 35 contains a massless Goldstone mode associated with the spatial fluctuations in the asymptotic long-time (and long-time-difference) limit. This means that correlation functions such as those in Eq. (37) are described by algebraically decaying functions $\mathcal{F}\left(r_{1}, r_{2}, \ldots, r_{N}\right)$. The absence of a correlation length $\xi$ leads to the non-Gaussian behavior for quantities averaged over a finite volume, similarly to the studies in Refs. 37 and 38. Of course, one expects that universal behavior of the PDFs will occur only after coarse graining. Note however that it is the absence of a correlation scale that implies that the law of large numbers will not take effect as the box size increases. So the theory based on reparametrization invariance of the action gives rise to a nonGaussian distribution for coarse-grained $C_{r}\left(t, t_{w}\right)$ (in addition to any coarse graining needed to define a theory in the space continuum) because of the algebraic correlations originating from the massless Goldstone mode. In the theory of 
Refs. 20, 21, and 35 we argued that the correlation scale $\xi \rightarrow \infty$ in the asymptotic long-time limit. However, for finite times there is a finite $\xi .{ }^{21}$ Coarse graining much beyond this length scale leads to Gaussian distributions.

The second element that the analytical theory contains is that all dependencies on the times are functions of $\Delta s$ $=s(t)-s\left(t_{w}\right)$. This leads to the collapse of distributions (not only their bulk averages) as a function of $\ln h_{S}(t) / h_{S}\left(t_{w}\right)$. In addition, the fact that the different $n=\Delta s / \delta s$ slice contributions $\dot{\psi}_{r}^{2}\left(s_{i}\right)$ are uncorrelated, and that they enter in the local correlations $C_{r}\left(t, t_{w}\right)$ through the sum over all slices, is the reason why the skewness of the distributions depend on $n$, as shown in Fig. 9. Again, this is in good accord with the experimental observations of Ref. 10 and with the numerical results for the models that we study in this paper.

In summary, we believe that the qualitative features shared by the PDFs of local correlators in the facilitated models, in the $3 d$ EA model, and in the experimental observations of Cipelletti et al. ${ }^{10}$ can be understood as a consequence of certain spatial and temporal correlations of the $C_{r}\left(t, t_{w}\right)$, which are contained in the theory based on reparametrization invariance.

\section{CONCLUSIONS}

In equilibrium and away from criticality any global observable of a macroscopic system has Gaussian fluctuations. At criticality, instead, one observes non-Gaussian fluctuations due to the divergence of the correlation length and the nonapplicability of the central limit theorem. Still, scale invariance at the critical point constrains the possible probability distributions; these are determined by the universality class to which the systems belong. A similar criterion to classify the probability distributions of the fluctuations of macroscopic observables in critical nonequilibrium systems is based on the use of symmetries. It has been proposed in Ref. 37.

The glass transition, where the system falls out of equilibrium, is a dynamic crossover and neither a dynamic nor a thermodynamic transition. However, many features of glassy dynamics resemble critical relaxation such as the fact that correlations do not decay exponentially, but with much slower decay forms. One could expect then that some concepts that have been useful to study critical phenomena could also apply to the glassy dynamics. ${ }^{41,42}$

In this paper we continued the study of local fluctuations in the dynamics of glassy systems. On the theoretical side we improved the $\sigma$ model, or random manifold action, proposed in Refs. 20, 21, and 35 to capture not only the scaling in time of the PDFs but also their functional form. On the numerical side, we showed that part of the predictions of the theory tested in Refs. 20 and 21 using the $3 d$ Edwards-Anderson model also hold for a nondisordered kinetically constrained spin model of a glass. This confirms that the existence of quenched disorder is not important in this respect and that kinetic frustration and energetic frustration lead to very similar nonequilibrium dynamics. Moreover, we tested the evolution of the form of the PDFs for these two models and verified that they behave in a way that resembles strongly the experimental observations in Ref. 10.

One could expect that a more detailed and extended analysis of the local dynamics of glassy systems might lead to better differentiation between models and systems. For instance, one could find that the simple action (35) is not enough to reproduce the special form of PDFs found in a system and hence be forced to include other terms that we here neglected.

\section{ACKNOWLEDGMENTS}

This work was supported in part by the NSF Grant Nos. DMR-0305482 and INT-0128922 (C.C.), a CNRS-NSF collaboration grant, an ACI Jeunes Chercheurs "Algorithmes d'optimization et systèmes désordonnés quantiques," the Guggenheim Foundation (LFC), FQRNT (PC), and the NSF Grant Nos. PHY99-07949 (C. C. L. F. C. D. R.) and CHE0134969 (P. C. D. R.). C. C. L. F. C. and D. R. thank the KITP Santa Barbara, and L. F. C. the Condensed Matter group at the Abdus Salam International Center for Theoretical Physics for their hospitality during part of the preparation of this work. We especially thank H. E. Castillo, S. Franz, M. P. Kennett, and J. Polchinski for very useful discussions. L.F.C. is a research associate at the Abdus Salam ICTP, Trieste, Italy.

\section{APPENDIX A: GENERALIZED GUMBEL DISTRIBUTIONS}

The Gumbel distribution is defined as

$$
\Phi_{a}(y)=\frac{|\alpha| a^{a}}{\Gamma(a)} e^{a\left[\alpha\left(y-y_{0}\right)-e^{\alpha\left(y-y_{0}\right)}\right]},
$$

where $\Gamma(a)$ is the gamma function. The parameters $y_{0}$ and $\alpha$ control the position of the center and the width of the distribution, respectively. One could define standard distributions by requiring that the center is at zero, and the width is unity. These conditions fix, for a given $a, y_{0}(a)$ and $\alpha(a)$ as

$$
\alpha=\sqrt{\Psi^{\prime}(a)}, \quad \alpha y_{0}=\ln a-\Psi(a),
$$

where $\Psi(a)$ is the digamma function $\Psi(a)=\Gamma^{\prime}(a) / \Gamma(a)$.

The Gumbel distribution with $a=1$ appears as one kind of asymptotic extreme value statistics when searching for the distribution of the maximum (or the minimum) of a sequence of independent identically distributed random variables with a probability density decaying faster than any power law. Extensions with integer parameter $a>1$ are the results of searching for the distribution of the $a$ th largest (smallest) value in the sequence. For any $a$, there is a choice of sign for $\alpha$ and the two signs correspond to the Gumbel statistics of either extreme minima or extreme maxima. Further extensions with noninteger parameter $a<1$ have been found when the elements of the sequence are correlated over a distance $1 / a{ }^{57}$ For $a=\pi / 2$ one recovers the distribution studied by Bramwell, Holdsworth, and Pinton. ${ }^{58}$

Finally, let us study two limits of the generalized extreme value distribution (A1). A Gaussian distribution of unit variance is obtained by taking the $a \rightarrow \infty$ limit of $\Phi_{a}(y)$ while holding the product $\alpha^{2} a=1$ fixed: 


$$
\begin{aligned}
& \lim _{\substack{a \rightarrow \infty, \alpha^{2} a=1\\
}} \Phi_{a}(y) \\
& =\lim _{a \rightarrow \infty, \alpha^{2} a=1} \frac{a^{a}}{\sqrt{a} \Gamma(a)} \\
& \quad \times e^{a\left[\alpha\left(y-y_{0}\right)-1-\alpha\left(y-y_{0}\right)-1 / 2 \alpha^{2}\left(y-y_{0}\right)^{2}+\mathcal{O}\left(\alpha^{3}\right)\right]} \\
& =\lim _{a \rightarrow \infty} \frac{a^{a} e^{-a}}{\sqrt{a} \Gamma(a)} e^{-1 / 2\left(y-y_{0}\right)^{2}}=\frac{1}{\sqrt{2 \pi}} e^{-1 / 2\left(y-y_{0}\right)^{2}},
\end{aligned}
$$

where in the last step we used Stirling's formula to get the normalization factor.

Another interesting limit is when $a \rightarrow 0$ while holding $|\alpha| a=1$ :

$$
\begin{aligned}
& \lim _{a \rightarrow 0,|\alpha| a=1} \Phi_{a}(y) \\
& =\lim _{a \rightarrow 0} \frac{a^{a}}{a \Gamma(a)} e^{\operatorname{sgn} \alpha\left(y-y_{0}\right)} \theta\left[-\operatorname{sgn} \alpha\left(y-y_{0}\right)\right] \\
& =e^{\operatorname{sgn} \alpha\left(y-y_{0}\right)} \theta\left[-\operatorname{sgn} \alpha\left(y-y_{0}\right)\right]
\end{aligned}
$$

where we used that $\lim _{a \rightarrow 0} a \Gamma(a)=\Gamma(1)=1$. Thus, the $a$ $\rightarrow 0$ limit leads to exponential distributions either starting or ending at $y=y_{0}$.

\section{APPENDIX B: INTRINSIC SKEWNESS OF UNCORRELATED DYNAMICS}

The probability distribution of local correlation functions evaluated at finite times and coarse-grained over a sufficiently large cells is, to a first approximation, normally distributed around the bulk value. However, for the times and cell sizes considered in this paper, one needs to consider how the intrinsic distribution of unconstrained spins contributes to the skewing of the distribution of local correlations. In this Appendix, we consider the degree of intrinsic skewing that arises from uncorrelated dynamics on a lattice.

To differentiate between correlated and intrinsic skewness, we look at a model of paramagnetism without any facilitation. The Hamiltonian is then the same as in the FA model. This toy model has no dependence on the dimensionality or the neighboring environment, and depends only on the number of spins included in the cells. Therefore, all sites are independent and the probability $p$ that an initially up spin is down at time $t$ is the same everywhere ( $q$ is the equivalent probability for the spins initially down). Using Metropolis Monte Carlo single spin flips, one finds the following distribution for a cell of $N$ spins using binomial distribution arguments,

$$
\begin{aligned}
\mathcal{P}(n, d, u)= & \frac{N !}{(n-d) ! d !(N-n-u) ! u !} c^{n}(1-c)^{N-n} \\
& \times p^{d}(1-p)^{n-d} q^{u}(1-q)^{N-n-u} .
\end{aligned}
$$

Here $n$ is the number of spins up initially up, $c$ is the initial bulk concentration of up spins, $d$ the number of initially up
TABLE I. Unconstrained parameters for a decay of the global correlations to a value of 0.7 of the initial value for the FA- $2 d 2 n$ model for a quenching $T=0.3$ and a box size of $N=10 \times 10$. The parameters are the $t_{w}$ defect concentration $c$, the concentration scaling factor $X$ for time $t$, and the probability $p$ of defect disappearance at time $t$. The resulting approximative Gumbel $a$ parameter is also given.

\begin{tabular}{llllll}
\hline \hline$t_{w}$ & $10^{1}$ & $10^{2}$ & $10^{3}$ & $10^{4}$ & $10^{5}$ \\
$t$ & 30 & 45 & 90 & 330 & 1000 \\
\hline$c$ & 0.30865 & 0.27257 & 0.21203 & 0.15505 & 0.1685 \\
$X$ & 0.938 & 0.969 & 0.988 & 0.995 & 0.9995 \\
$p$ & 0.233 & 0.230 & 0.237 & 0.258 & 0.264 \\
$a$ & 100 & $\sim \infty$ & $\sim \infty$ & $\sim \infty$ & $\sim \infty$ \\
\hline \hline
\end{tabular}

spins that are down at time $t$, and $u$ of initially down spins which are up at time $t$. In the equilibrium case, $p$ and $q$ are related by

$$
q_{\text {eq }}=c_{\text {eq }} /\left(1-c_{\text {eq }}\right) p_{\text {eq }}
$$

The same relationship (without the subscripts) holds for a constant concentration system, but not in the case of a steadily decreasing concentration, as in the quenched facilitated models. That, however, can be resolved by including a scaling factor $X$ proportional to the concentration during the quench as a function of $t_{w}$ and $t$.

For a given ensemble average value $\mathcal{C}$ of the single site correlation, one can obtain the PDF for the correlation averaged over a number $V_{r}=\ell^{d}$ of uncorrelated spins. (Notice that, since these are free spins without any dynamical constraint, the number of time steps necessary to reach the average value $\mathcal{C}$ is much smaller than that for the kinetically constrained models.) Using the result in (B1), one can obtain the corresponding value of the cell connected correlation function

$$
C_{c, \text { norm }}(t) \equiv \overline{c(0) c(t)}-\overline{c(0) c(t)}
$$

where $\overline{c(t)}=n-d-u / N, \quad \overline{c(0)}=n / N, \quad$ and $\overline{c(0) c(t)}=n$ $-d / N$. The overline refers to the subensemble average.

Using bulk values for the different parameters, the statistical component of the distribution of the local spin correlation can be obtained, by numerically evaluating Eq. (B3) for all $n, u$, and $d$ and properly weighting them according to Eq. (B1). Tables I, II, and III show the parameters used in the different models. In the case of the plaquette models, the same discussion applies, but with a null magnetic field instead.

TABLE II. Unconstrained parameters for a decay of the global correlations to a value of 0.7 of the initial value for the FA- $3 d 3 n$ model for a quenching $T=0.4$ and a box size of $N=5 \times 5 \times 5$. Parameter definitions are identical to those in Table I.

\begin{tabular}{lccccc}
\hline \hline$t_{w}$ & $10^{1}$ & $10^{2}$ & $10^{3}$ & $10^{4}$ & $10^{5}$ \\
$t$ & 15 & 26 & 81 & 370 & 2300 \\
\hline$c$ & 0.3299 & 0.2903 & 0.2399 & 0.2007 & 0.1685 \\
$X$ & 0.959 & 0.982 & 0.993 & 0.996 & 0.998 \\
$p$ & 0.220 & 0.219 & 0.2305 & 0.241 & 0.249 \\
$a$ & 80 & 80 & 100 & $\sim \infty$ & $\sim \infty$ \\
\hline \hline
\end{tabular}


TABLE III. Unconstrained parameters for a decay of the global correlations to a value of 0.88 of the initial value for the FA- $3 d 3 n$ model for a quenching $T=0.4$ and a box size of $N=5 \times 5 \times 5$. Parameter definitions are identical to those in Table I.

\begin{tabular}{lccccc}
\hline \hline$t_{w}$ & $10^{1}$ & $10^{2}$ & $10^{3}$ & $10^{4}$ & $10^{5}$ \\
$t$ & 2 & 4 & 10 & 32 & 100 \\
\hline$c$ & 0.3299 & 0.2903 & 0.2399 & 0.2007 & 0.1685 \\
$X$ & 0.992 & 0.997 & 0.9996 & 1.000 & 1.000 \\
$p$ & 0.0812 & 0.0908 & 0.0902 & 0.0962 & 0.0993 \\
$a$ & 15 & 20 & 40 & 90 & 100 \\
\hline \hline
\end{tabular}

In the first two cases (Tables I and II) the bulk correlation is chosen to have a rather large value and the Gumbel parameters found are extremely large corresponding to approximately Gaussian distributions. No interesting effect is observed in these cases. In the last example considered (Table III) the bulk correlation is chosen to take a smaller value and the parameters found indicate that the distribution is skewed. However, if one compares to the results obtained for the same model with facilitation one concludes that the non-Gaussian effect is here much weaker and tends to disappear rather quickly when the times involved get long.

In summary, the simple correlations that follow from coarse graining of some binomially distributed single-site spin-spin correlation whose average is restricted to be $\mathcal{C}$ cannot account for the PDFs we find for the kinetically constrained model. Spatial correlations between the spin variables are needed to account for both the width and skeweness of the distributions.

${ }^{1}$ M. D. Ediger, C. A. Angell, and S. R. Nagel, J. Phys. Chem. 100, 13200 (1996).

${ }^{2}$ H. Sillescu, J. Non-Cryst. Solids 243, 81 (1999); M. D. Ediger, Annu. Rev. Phys. Chem. 51, 99 (2000).

${ }^{3}$ W. K. Kegel and A. V. Blaaderen, Science 287, 290 (2000).

${ }^{4}$ E. Weeks, J. C. Crocker, A. C. Levitt, A. Schofield, and D. A. Weitz, Science 287, 627 (2000).

${ }^{5}$ E. R. Weeks and D. A. Weitz, Phys. Rev. Lett. 89, 095704 (2002).

${ }^{6}$ R. E. Courtland and E. R. Weeks, J. Phys.: Condens. Matter 15, S359 (2003).

${ }^{7}$ E. Vidal Russell, N. E. Israeloff, L. E. Walther, and H. Alvarez Gomariz, Phys. Rev. Lett. 81, 1461 (1998); L. E. Walther, N. E. Israeloff, E. Vidal Russell, and H. Alvarez Gomariz, Phys. Rev. B 57, R15112 (1998); E. Vidal Russel and N. E. Israeloff, Nature (London) 408, 695 (2000).

${ }^{8}$ R. S. Miller and R. A. MacPhail, J. Phys. Chem. 101, 8635 (1997).

${ }^{9}$ L. Buisson, L. Bellon, and S. Ciliberto, cond-mat/0210490.

${ }^{10}$ L. Cipelletti, H. Bissig, V. Trappe, P. Ballestat, and S. Mazoyer, J. Phys.: Condens. Matter 15, S257 (2003); H. Bissig, V. Trappe, S. Romer, and L. Cipelletti, cond-mat/0301265.

${ }^{11}$ B. B. Laird and H. R. Schober, Phys. Rev. Lett. 66, 636 (1991); H. R. Schober and B. B. Laird, Phys. Rev. B 44, 6746 (1991).

${ }^{12}$ T. Muranaka and Y. Hiwatari, Phys. Rev. E 51, R2735 (1995); M. M. Hurley and P. Harrowell, ibid. 52, 1694 (1995); D. N. Perera and P. Harrowell, ibid. 59, 5721 (1999).

${ }^{13}$ W. Kob, C. Donati, S. J. Plimpton, P. H. Poole, and S. C. Glotzer, Phys. Rev. Lett. 79, 2827 (1997); C. Donati, J. F. Douglas, W. Kob, S. J. Plimpton, P. H. Poole, and S. C. Glotzer, ibid. 80, 2338 (1998); C. Donati, S. C. Glotzer, P. H. Poole, W. Kob, and S. J. Plimpton, Phys. Rev. E 60, 3107 (1999).

${ }^{14}$ A. Heuer and A. Okun, J. Chem. Phys. 106, 6176 (1997); B. Doliwa and A. Heuer, Phys. Rev. Lett. 80, 4915 (1998).

${ }^{15}$ G. Johnson, A. I. Mel'cuk, H. Gould, W. Klein, and R. D. Mountain, Phys. Rev. E 57, 5707 (1998).

${ }^{16}$ R. Yamamoto and A. Onuki, Phys. Rev. E 58, 3515 (1998).
${ }^{17}$ F. W. Starr, S. Sastry, J. F. Douglas, and S. C. Glotzer, Phys. Rev. Lett. 89, 125501 (2002).

${ }^{18}$ C. Oglichler and H. R. Schober, Phys. Rev. B 59, 811 (1999).

${ }^{19}$ K. Vollmayr-Lee, W. Kob, K. Binder, and A. Zippelius, J. Chem. Phys. 116, 5158 (2002).

${ }^{20}$ H. Castillo, C. Chamon, L. F. Cugliandolo, and M. P. Kennett, Phys. Rev. Lett. 88, 237201 (2002).

${ }^{21}$ H. Castillo, C. Chamon, L. F. Cugliandolo, J. L. Iguain, and M. P. Kennett, Phys. Rev. B 68, 134442 (2003).

${ }^{22}$ J.-P. Bouchaud, L. F. Cugliandolo, J. Kurchan, and M. Mézard, in SpinGlasses and Random Fields, edited by A. P. Young (World Scientific, Singapore, 1998).

${ }^{23}$ L. F. Cugliandolo, in Slow Relaxations and Nonequilibrium Dynamics in Condensed Matter, edited by J.-L. Barrat et al. (Springer, Berlin, 2002).

${ }^{24}$ L. F. Cugliandolo and J. Kurchan, Phys. Rev. Lett. 71, 173 (1993).

${ }^{25}$ J.-P. Bouchaud, L. F. Cugliandolo, J. Kurchan, and M. Mézard, Physica A 226, 243 (1996)

${ }^{26}$ J. Kurchan and L. Laloux, J. Phys. A 29, 1929 (1996); A. Cavagna, Europhys. Lett. 53, 490 (2001).

${ }^{27}$ L. Angelani, G. Ruocco, M. Sampoli, and F. Sciortino, J. Chem. Phys. 119, 2120 (2003); A. Crisanti and F. Ritort, Europhys. Lett. 52, 640 (2000).

${ }^{28}$ G. H. Frederickson and H. C. Andersen, Phys. Rev. Lett. 53, 1244 (1984); G. H. Frederickson and H. C. Andersen, J. Chem. Phys. 83, 5822 (1985); G. H. Fredrickson and S. A. Brawer, ibid. 84, 3351 (1986).

${ }^{29}$ W. Kob and H. C. Andersen, Phys. Rev. E 48, 4364 (1993).

${ }^{30}$ J. Jäckle and A. Krönig, J. Phys.: Condens. Matter 6, 7633 (1994); J. Jäckle, ibid. 14, 1423 (2002).

${ }^{31}$ J. Kurchan, L. Peliti, and M. Sellitto, Europhys. Lett. 39, 365 (1997); M. Sellitto, Eur. Phys. J. B 4, 135 (1998); M. Sellitto, J. Phys.: Condens. Matter 14, 1455 (2002).

${ }^{32}$ F. Ritort and P. Sollich, Adv. Phys. 52, 219 (2003).

${ }^{33}$ S. Butler and P. Harrowell, J. Chem. Phys. 95, 4454 (1991); 95, 4466 (1991); P. Harrowell, Phys. Rev. E 48, 4359 (1993); M. Foley and P. Harrowell, J. Chem. Phys. 98, 5069 (1993).

${ }^{34}$ J. P. Garrahan and D. Chandler, Phys. Rev. Lett. 89, 035704 (2002); L. Berthier and J. P. Garrahan, Phys. Rev. E 68, 041201 (2003); J. P. Garrahan and D. Chandler, Proc. Natl. Acad. Sci. U.S.A. 100, 9710 (2003).

${ }^{35}$ C. Chamon, M. P. Kennett, H. E. Castillo, and L. F. Cugliandolo, Phys. Rev. Lett. 89, 217201 (2002).

${ }^{36}$ N. D. Goldenfeld, Lectures on Phase Transitions and the Renormalisation Group (Addison-Wesley, Reading, MA, 1992).

${ }^{37}$ Z. Rácz, in Slow Relaxations and Nonequilibrium Dynamics in Condensed Matter, edited by J.-L. Barrat et al. (Springer, 2002); G. Gyögyi, P. C. W. Holdsworth, B. Portelli, and Z. Rácz, Phys. Rev. E 68, 056116 (2003); T. Antal, M. Droz, G. György, and Z. Rácz, Phys. Rev. Lett. 87, 240601 (2001); G. Foltin, K. Oerding, Z. Rácz, R. L. Workman, and R. K. P. Zia, Phys. Rev. E 50, 639 (1994).

${ }^{38}$ S. T. Bramwell, J. Y. Fortin, P. C. W. Holdsworth, S. Peysson, J. F. Pinton, B. Portelli, and M. Sellitto, Phys. Rev. E 63, 041106 (2001); B. Portelli, P. C. W. Holdsworth, M. Sellitto, and S. T. Bramwell, ibid. 64, 036111 (2001).

${ }^{39}$ N. Lačević, F. W. Starr, T. B. Schroeder, and S. C. Glotzer, J. Chem. Phys. 119, 7372 (2003); C. Bennemann, C. Donati, J. Baschnagel, and S. Glotzer, Nature (London) 399, 246 (1999).

${ }^{40}$ S. Franz, C. Donati, G. Parisi, and S. C. Glotzer, Philos. Mag. B 79, 1827 (1999). S. Franz and G. Parisi, J. Phys.: Condens. Matter 12, 6335 (2000).

${ }^{41}$ S. Whitelam, L. Berthier, and J. P. Garrahan, cond-mat/0310207.

${ }^{42}$ G. Biroli and J.-P. Bouchaud, cond-mat/0401260.

${ }^{43}$ I. S. Graham, L. Piché, and M. Grant, Phys. Rev. E 55, 2132 (1997).

${ }^{44}$ S. F. Edwards and P. W. Anderson, J. Phys. F: Met. Phys. 5, 965 (1975).

${ }^{45}$ D. Hérisson and M. Ocio, Phys. Rev. Lett. 88, 257202 (2002).

${ }^{46}$ B. Abou, D. Bonn, and J. Meunier, Phys. Rev. E 64, 021510 (2001); D. Bonn, S. Tanase, B. Abou, H. Tanaka, and J. Meunier, Phys. Rev. Lett. 89, 015701 (2002).

${ }^{47}$ A. Knaebel, M. Bellour, J.-P. Munch, V. Viasnoff, F. Lequeux, and J. L. Harden, Europhys. Lett. 52, 73 (2000); V. Viasnoff and F. Lequeux, Phys. Rev. Lett. 89, 065701 (2002).

${ }^{48}$ L. Cipelletti, S. Manley, R. C. Ball, and D. A. Weitz, Phys. Rev. Lett. 84, 2275 (2000); L. Ramos and L. Cipelletti, ibid. 87, 245503 (2001).

${ }^{49}$ O. Pouliquen, M. Belzons, and M. Nicolas, cond-mat/0305659.

${ }^{50}$ L. F. Cugliandolo and J. Kurchan, J. Phys. A 27, 5749 (1994).

${ }^{51}$ M. Picco, F. Ritort, and F. Ricci-Tersenghi, Eur. Phys. J. B 21, 211 (2001).

${ }^{52}$ G. Biroli, S. Franz, M. Sellitto, and C. Toninelli (unpublished). 
${ }^{53}$ C. Toninelli, G. Biroli, and D. S. Fisher, cond-mat/0306746.

${ }^{54}$ G. Parisi, in Slow Relaxations and Nonequilibrium Dynamics in Condensed Matter, edited by J.-L. Barrat et al. (Springer-Verlag Berlin, 2002); D. S. Fisher, in Slow Relaxations and Nonequilibrium Dynamics in Condensed Matter, edited by J.-L. Barrat et al. (Springer Berlin, 2002).

${ }^{55}$ E. Bertin and J.-P. Bouchaud, J. Phys. A 35, 3039 (2002).

${ }^{56}$ S. Franz and M. Mézard, Europhys. Lett. 26, 209 (1994); S. Franz and M. Mézard, Physica A 210, 48 (1994).
${ }^{57}$ E. J. Gumbel, Statistics of Extremes (Columbia University Press, New York, 1958).

${ }^{58}$ S. Bramwell, P. W. Holdsworth, and J.-F. Pinton, Nature (London) 396, 552 (1998); S. T. Bramwell, J. Y. Fortin, P. C. W. Holdsworth et al., Phys. Rev. Lett. 84, 3744 (2000).

${ }^{59}$ Note that $\dot{\phi}_{r}(s)$ is a positive definite quantity; indeed, $0 \leqslant \dot{\phi}_{r}(t)$ $=d \phi_{r}(t(s)) / d s d s / d t=\dot{\phi}_{r}(s) \dot{h}_{S}(t) / h_{S}(t)$ implies $\dot{\phi}_{r}(s) \geqslant 0$. 